\title{
Circadian clocks guide dendritic cells into skin lymphatics
}

\author{
Stephan J. Holtkamp', Louise M. Ince $\mathbb{B}^{2}{ }^{2}$, Coline Barnoud ${ }^{2}{ }^{2}$, Madeleine T. Schmitt ${ }^{1,3}$, \\ Flore Sinturel ${ }^{4,5,6,7}$, Violetta Pilorz ${ }^{8}$, Robert Pick ${ }^{2}{ }^{2}$, Stéphane Jemelin ${ }^{2}$, Michael Mühlstädt ${ }^{9}$, \\ Wolf-Henning Boehncke ${ }^{2,9}$, Jasmin Weber ${ }^{1}$, David Laubender ${ }^{10}$, Julia Philippou-Massier ${ }^{11}$, \\ Chien-Sin Chen ${ }^{1} 1$, Leonie Holtermann ${ }^{12}$, Dietmar Vestweber ${ }^{12}{ }^{12}$, Markus Sperandio ${ }^{1}$ ', \\ Barbara U. Schraml ${ }^{1}$, Cornelia Halin ${ }^{13}{ }^{13}$, Charna Dibner ${ }^{4,5,6,7}$, Henrik Oster ${ }^{8}$, Jörg Renkawitz ${ }^{1,3}$ and \\ Christoph Scheiermann $\mathbb{D}^{1,2}$ 不
}

\begin{abstract}
Migration of leukocytes from the skin to lymph nodes (LNs) via afferent lymphatic vessels (LVs) is pivotal for adaptive immune responses ${ }^{1,2}$. Circadian rhythms have emerged as important regulators of leukocyte trafficking to LNs via the blood $^{3,4}$. Here, we demonstrate that dendritic cells (DCs) have a circadian migration pattern into $L V s$, which peaks during the rest phase in mice. This migration pattern is determined by rhythmic gradients in the expression of the chemokine CCL21 and of adhesion molecules in both mice and humans. Chronopharmacological targeting of the involved factors abrogates circadian migration of DCs. We identify cell-intrinsic circadian oscillations in skin lymphatic endothelial cells (LECs) and DCs that cogovern these rhythms, as their genetic disruption in either cell type ablates circadian trafficking. These observations indicate that circadian clocks control the infiltration of DCs into skin lymphatics, a process that is essential for many adaptive immune responses and relevant for vaccination and immunotherapies.
\end{abstract}

Steady-state migration of dermal DCs into afferent LVs is tightly regulated by a variety of promigratory factors, including the CCL21-CCR7 chemokine axis and the adhesion molecules LYVE1, CD99 and JAM-A ${ }^{1,2,5-12}$. In the LN, 24-h-long circadian rhythms influence the homing capacity and function of lymphocytes ${ }^{4,13-16}$; however, whether the draining of leukocytes from tissues occurs in a rhythmic manner is unknown.

To address this question, we collected mouse ear skin at different times of the day (that is, zeitgeber time 1 (ZT1; $1 \mathrm{~h}$ after light onset in a 12-h light/12-h dark environment; 'morning'), ZT7 ('day'), ZT13 ('evening') and ZT19 ('night')) and cultured the explants for $6 \mathrm{~h}$ in medium. We then quantified the location of tissue-endogenous CD $11 c^{+}$DCs inside LYVE- ${ }^{+}$skin lymphatics by immunofluorescence imaging (Fig. 1a and Extended Data Fig. 1a,b). Infiltration of
CD11 ${ }^{+}$cells into the LVs peaked at ZT7 (day) and troughed at ZT19 (night) (Fig. 1a and Supplementary Table 1). Additional quantification of the location of CD $11 \mathrm{c}^{+}$DCs in the ear at steady state at ZT1, ZT7, ZT13 and ZT19 without ensuing culture confirmed a stronger intralymphatic presence of cells during the day than at night (Extended Data Fig. 1c). Explants that were collected at ZT7 and ZT19 and cultured for $24 \mathrm{~h}$ still exhibited higher CD11 $\mathrm{c}^{+}$infiltration in LVs at ZT7 than at ZT19 (Fig. 1b and Extended Data Fig. 1b), indicating that night migration did not catch up to the day migration. The diurnal migration of $\mathrm{CD}_{11 \mathrm{c}^{+}} \mathrm{DCs}$ into LVs was also detected after the topical application of fluorescein isothiocyanate (FITC), an inflammatory stimulus (Fig. 1c), indicating that the migration differences were maintained during inflammation. The amount of cells analyzed and the overall LV volume was similar at all time points (Extended Data Fig. 1d,e).

Circadian rhythms are defined by their persistence in the absence of external entraining factors, such as rhythmic light onset and offset. To investigate whether the oscillations in DC migration into LVs were circadian in nature, mice were housed in constant darkness. The migration differences of CD11 $\mathrm{c}^{+} \mathrm{DCs}$ in LVs between the peak at ZT7 (day) and the trough at ZT19 (night) continued in conditions of constant darkness, demonstrating these oscillations to be bona fide circadian (Fig. 1d). The oscillations adjusted to a 12-h inverted light-dark cycle (Fig. 1d), indicating that they could be phase shifted and entrained by light, an additional feature of circadian rhythms. This indicated that $\mathrm{CD} 11 \mathrm{c}^{+} \mathrm{DC}$ migration into skin LVs was driven by endogenous circadian rhythms and did not represent solely a response to a rhythmic day-night environment.

In situ whole-mount staining of earsidentified that $\mathrm{CD} 1 \mathrm{c}^{+}$langerin $\mathrm{CD} 103^{-}$dermal conventional DCs (cDC2s) and CD11 ${ }^{+}$langerin ${ }^{+}$ Langerhans cells (LCs) preferentially migrated into LVs at ZT7 (day) compared to at ZT19 (night), while very few CD11 ${ }^{+}$langerin ${ }^{-} \mathrm{CD} 103^{+}$

'Biomedical Center (BMC), Institute for Cardiovascular Physiology and Pathophysiology, Walter Brendel Center for Experimental Medicine (WBex), Faculty of Medicine, Ludwig-Maximilians-Universität Munich, Planegg-Martinsried, Germany. ${ }^{2}$ Department of Pathology and Immunology, Faculty of Medicine, University of Geneva, Geneva, Switzerland. 'Laboratory 'Cell Biology of the Immune System', Biomedical Center (BMC), Institute for Cardiovascular Physiology and Pathophysiology, Walter Brendel Center for Experimental Medicine (WBex), Faculty of Medicine, Ludwig-Maximilians-Universität Munich, Planegg-Martinsried, Germany. ${ }^{4}$ Department of Medicine, Division of Endocrinology, Diabetes, Nutrition and Patient Education, University Hospitals of Geneva, Geneva, Switzerland. ${ }^{5}$ Department of Cell Physiology and Metabolism, University of Geneva, Geneva, Switzerland. ${ }^{6}$ Diabetes Center, Faculty of Medicine, University of Geneva, Geneva, Switzerland. 'Institute of Genetics and Genomics of Geneva (iGE3), University of Geneva, Geneva, Switzerland. ${ }^{8}$ Institute of Neurobiology, University of Lübeck, Lübeck, Germany. ${ }^{9}$ Division of Dermatology and Venereology, Department of Medicine, University Hospitals of Geneva, Geneva, Switzerland. ${ }^{10}$ Max Planck Institute of Neurobiology, Martinsried, Germany. "Laboratory for Functional Genome Analysis, Gene Center Munich, Ludwig-Maximilians-Universität Munich, Munich, Germany. ${ }^{2}$ Department of Vascular Cell Biology, Max Planck Institute for Molecular Biomedicine, Münster, Germany. ${ }^{13}$ nstitute of Pharmaceutical Sciences, ETH Zurich, Zurich, Switzerland. ${ }^{凶 e}$-mail: christoph.scheiermann@unige.ch 
a

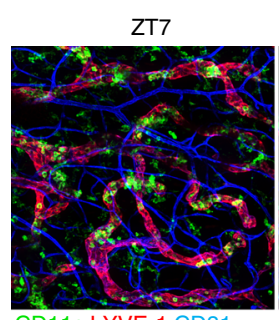

CD11c LYVE-1 CD31

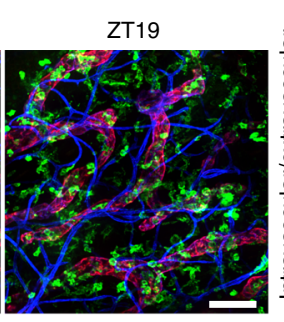

g

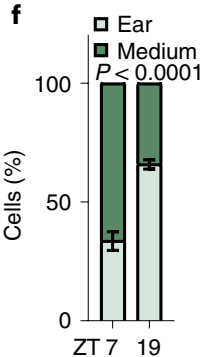

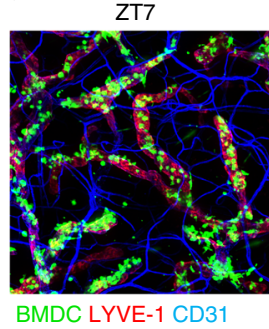

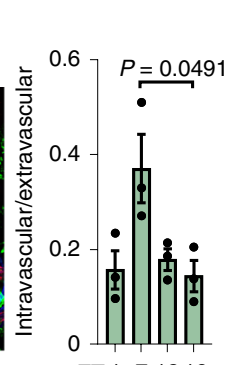

ZT 171319 b

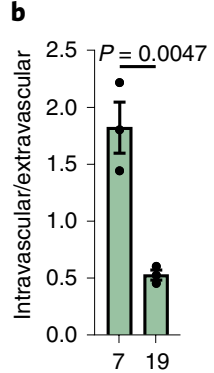

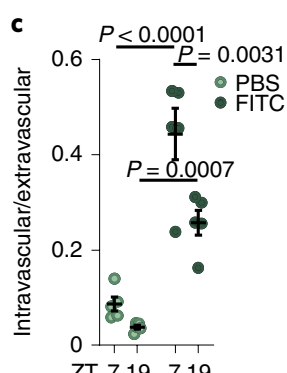

ZT 719719
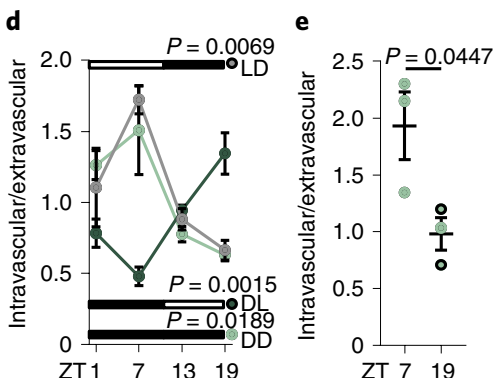
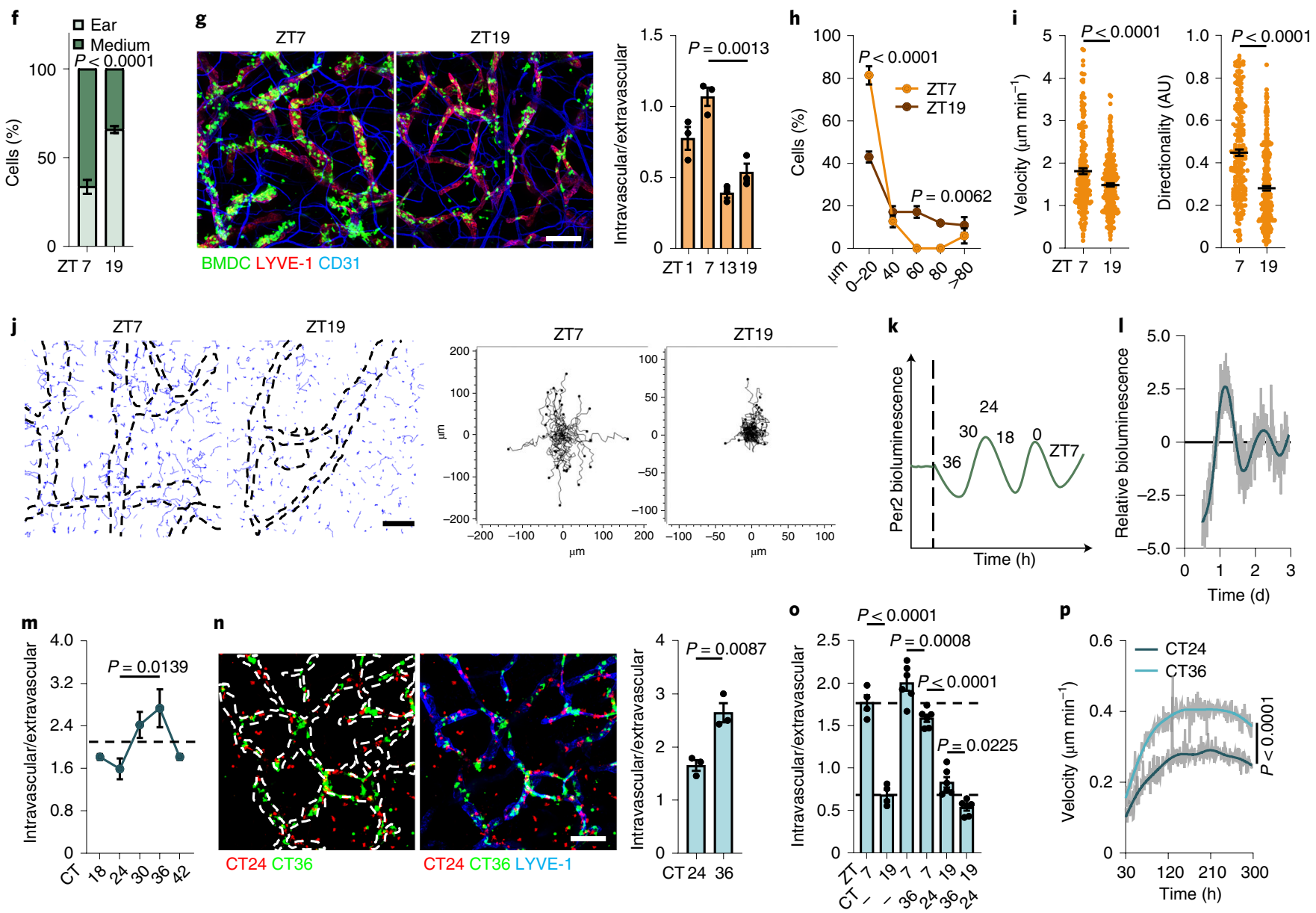

Fig. 1 Migration of dermal DCs into skin lymphatics is circadian. a,b, Crawl-in assays of ear CD11c ${ }^{+}$cells in LVs after $6 \mathrm{~h}$ (a) or $24 \mathrm{~h}$ (b); $n=3$ mice, one-way analysis of variance (ANOVA) with Tukey's post test (a) and unpaired Student's t-test (b). c, Ear CD11c $c^{+}$DCs in LVs after 24-h FITC painting; $n=5$ mice from two independent experiments; two-way ANOVA with Sidak post test. d, Ear CD11c cells in LVs in light:dark (LD), dark:light (DL) or constant darkness (DD) crawl-in assays; $n=3$ mice; one-way ANOVA. e, CD11c $c^{+}$langerin- DCs after ear crawl-in assays; $n=3$ mice; unpaired Student's $t$-test. f, Flow cytometry of ear and medium

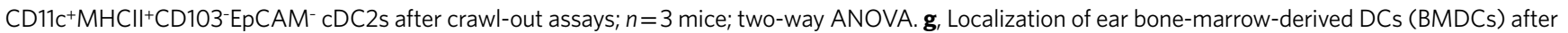
3-h crawl-in assays; $n=3$ mice; one-way ANOVA with Tukey's post test. $\mathbf{h}$, Localization of ear BMDCs after 1-h crawl-in assays; $n=4$ mice; unpaired Student's $t$-test. i, Velocity and directionality of BMDCs in ear crawl-in assays; $n=217$ and 301 cells, respectively, from four mice from four independent experiments; unpaired Student's t-test; AU, arbitrary units. j, Migration tracks (blue, left) of BMDCs overlaid onto LVs (dotted lines, left) and coordinates (right); $n=34$ cells from four mice from four independent experiments. $\mathbf{k}$, BMDC synchronization with serum at different circadian times (CTs). I, Bioluminescence oscillations from lipopolysaccharide (LPS)-activated Per2:Luc BMDCs after synchronization with serum. $\mathbf{m}$, Synchronized BMDCs after 3-h ear crawl-in assays (non-synchronized control is represented by dotted lines). CT18/CT42 is double plotted to facilitate viewing; $n=4$ mice; one-way ANOVA with Tukey's post test. $\mathbf{n}$, Localization of CT24 and CT36 synchronized BMDCs after crawl-in assays; $n=4$ mice; unpaired Student's $t$-test. o, Localization of CT24 and CT36 synchronized BMDCs after crawl-in assays; $n=4$ non-synchronized and $n=6$ synchronized BMDCs; one-way ANOVA with Tukey's post test. $\mathbf{p}$, Velocity of synchronized BMDCs in collagen migration assays; $n=3$ mice; Kruskal-Wallis test with Dunn's post test. Scale bars, $100 \mu \mathrm{m}$. Data are representative of at least two independent experiments (a, $\mathbf{b}$, $\mathbf{d}-\mathbf{h}, \mathbf{m}-\mathbf{p})$. All data are represented as mean \pm s.e.m.

cDC1s were detected (Fig. 1e and Extended Data Fig. 1f,g). Flow cytometry of the $\mathrm{CD} 11 \mathrm{c}^{+} \mathrm{MHCII}{ }^{\mathrm{hi}}$ cells that emigrated from the skin to the culture medium at ZT7 and ZT19 (Fig. 1f and Extended
Data Fig. 1h-j) further indicated that dermal CD103-EpCAM- DCs and $\mathrm{CD}_{103}$ EpCAM $^{+}$LCs emigrated from the skin in a highly time-of-day-dependent manner, peaking at ZT7 (day). 
To tease apart the role of the microenvironment from cell-autonomous processes, batches of LPS-activated BMDCs ${ }^{17}$ were generated in vitro. Circadian clocks are desynchronized in culture, and thus BMDCs have no circadian rhythm at the population level ${ }^{18}$. BMDCs were added for $3 \mathrm{~h}$ onto split ears collected at ZT1, ZT7, ZT13 and ZT19 from mice housed in light cabinets exhibiting lighting regimens timed for a 6-h difference from each other, enabling the simultaneous collection of ears at four different times of the day (Extended Data Fig. 2a). Using quantitative immunofluorescence imaging assays, we found elevated numbers of labeled leukocytes in the lymphatic capillaries of ZT7 (day) ears compared to ZT19 (night) ears (Fig. 1 g and Extended Data Fig. 2b,c). The number of transferred BMDCs present in the skin explants and the LV volume did not differ between time points (Extended Data Fig. 2d). When the BMDC-skin explant culture time (migration time) was shortened to 10-60 min, we detected the accumulation of BMDCs in close proximity to LVs only in the ZT7 (day) ears (Fig.1h and Extended Data Fig. 2e), indicating that diurnal migration differences occur early on. In addition, live imaging of the skin explants showed that BMDCs added onto ZT7 (day) ears exhibited higher velocity and directionality as well as increased total and Euclidean distance compared to BMDCs added to ZT19 (night) ears (Fig. 1i,j, Extended Data Fig. 2f,g and Supplementary Videos 1 and 2). These observations indicated that the ear microenvironment governed the migration capacity of BMDCs.

To assess the influence of circadian rhythms in DCs, LPS-activated BMDCs derived from mice expressing a fusion protein of the circadian protein PER2 and luciferase (PER2-Luc) ${ }^{19}$ were treated with

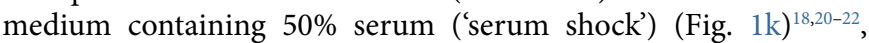
which synchronized PER2-Luc expression (Fig. 11) without affecting cell viability (Extended Data Fig. 2h). When BMDCs collected $24 \mathrm{~h}$ and $36 \mathrm{~h}$ after synchronization (CT24 and CT36, respectively) were added onto split ears from ZT7 mice, PER2-Luc ${ }^{\text {lo }}$ CT36 BMDCs (day BMDCs) migrated more efficiently into the afferent lymphatics of skin explants than PER2-Luc ${ }^{\text {hi }}$ CT24 BMDCs (night BMDCs) (Fig. 1m). The same results were observed when PER2Luc $^{\text {lo }}$ CT36 synchronized and PER2-Luch CT24 synchronized BMDCs were added together onto the same ZT7 ears (Fig. 1n and Supplementary Video 3), indicating that rhythmicity of migration into LVs was due to a DC-intrinsic mechanism. Using day (PER2Luc $^{\text {lo }}$ CT36) BMDCs on day ears (ZT7) showed maximal cell infiltration into LVs, while night (PER2-Luc ${ }^{\text {hi }}$ CT24) BMDCs on night ears (ZT19) showed minimal cell infiltration (Fig. 10). Day (PER2Luc $^{\text {lo }}$ CT36) BMDCs on night (ZT19) ears as well as night (PER2Luc $^{\text {hi }}$ CT24) BMDCs on day ears (ZT7) resulted in an intermediate capacity of BMDCs to immigrate into LVs (Fig. 1o), demonstrating that in-phase synchronization of both cells and environment provided maximal and minimal migration capacities, respectively. Synchronized PER2-Luc ${ }^{\text {lo }}$ CT36 (the time of maximal day migration capacity) BMDCs migrated better than unsynchronized BMDCs (Fig. 1m,o), indicating a benefit of rhythms for overall DC migration behavior. In addition, PER2-Luc ${ }^{\text {lo }}$ CT36 day BMDCs migrated with a higher velocity than PER2-Luc ${ }^{\text {hi }}$ CT24 night BMDCs in a collagen gel chemotaxis assay (Fig. 1p). These observations indicate that dermal DC migration into afferent lymphatics is controlled by both microenvironmental and DC-autonomous mechanisms.

We next used quantitative in situ imaging approaches to assess whether the signals involved in DC trafficking to the LVs oscillated in LECs (Extended Data Fig. 3a,b). Afferent LECs in epithelial barrier organs, such as skin, lung and gut but also in LNs, had a tissue-specific temporal expression profile of adhesion molecules (Fig. 2a, Extended Data Fig. 3c-g and Supplementary Table 2). In the skin, we observed strong oscillations of LYVE-1, CD99, JAM-A and JAM-C, molecules that have been implicated in steady-state DC migration $^{23}$, while other adhesion molecules were present but not oscillatory (such as VE-cadherin and PECAM-1) or not expressed at all (such as VCAM-1 and ICAM-1; Fig. 2a,b and Extended Data Fig. 3g). Integrating the profiles from all signals across all organs over time revealed a peak in the expression of oscillatory signals during the day (Fig. 2c), indicating that afferent LECs in the assessed organs have a higher leukocyte recruitment capacity at this time. Of relevance, we detected an analogous rhythm in the expression patterns of LYVE-1 and JAM-A in human skin biopsies (Fig. 2d and Extended Data Fig. 3h). In contrast to murine skin, human dermal LVs showed a nadir in the expression of migratory signals around 12:00 (Fig. 2d and Extended Data Fig. 3h), suggesting that in mice and humans, these signals exhibit a trough during the active phase of these organisms.

Quantitative real-time PCR (qPCR) analyses of sorted mouse dermal CD31 ${ }^{+}$podoplanin ${ }^{+}$LECs at ZT1, ZT7, ZT13 and ZT19 (Extended Data Fig. 4a) revealed a night peak in the mRNA amounts for Lyve1 and Cd99 (Fig. 2e and Extended Data Fig. 4b), indicating that these rhythms are under transcriptional control. To obtain unbiased insights into the global time-of-day-dependent changes within LECs, we performed bulk RNA sequencing analyses of sorted dermal CD31+ ${ }^{+}$odoplanin ${ }^{+}$LECs at ZT1, ZT7, ZT13 and ZT19 (Extended Data Fig. 4c). LECs exhibited strong oscillations in core components of the circadian clock, including the transcription factors Bmal1 and Per2 (Fig. 2f and Extended Data Fig. 4d). Moreover, GO enrichment analyses yielded a highly rhythmic cellular profile, particularly for adhesion processes (GO:0022610) (Fig. $2 \mathrm{~g}-\mathrm{i}$ and Supplementary Table 3). Flow cytometry on the culture medium of ear explants at ZT7 (day) and ZT19 (night) indicated that $\mathrm{CD} 103^{-} \mathrm{EpCAM}^{-}$dermal cDCs and CD103-EpCAM ${ }^{+} \mathrm{LCs}$ exhibited elevated expression of CCR7 at ZT7 compared to ZT19 (Fig. $2 \mathrm{j}$ and Extended Data Fig. 4e,f), while Ccr7 mRNA in synchronized CT18, CT24, CT30 and CT36 BMDCs showed a circadian expression profile, peaking at CT24 (Fig. 2k). These observations indicated that LECs express a circadian clock machinery, and LECs and DCs express promigratory factors in a rhythmic fashion.

Because expression of the chemokine receptor CCR7 was rhythmic in DCs, we investigated the expression of CCL21, the CCR7 ligand, in LECs. In quantitative immunofluorescence imaging analyses of the mouse ear, intracellular expression of CCL21 protein peaked at ZT7 in LECs (Fig. 3a,b). CCL21 also had a rhythmic expression in human skin biopsies, with a peak at 8:00 (Fig. 3b). Ccl21 mRNA abundance peaked at night (ZT19) in mouse LECs (Fig. 3c), indicating it was phase shifted compared to CCL21 protein and pointing to transcriptional control of its rhythmic expres$\operatorname{sion}^{24}$. Higher CCL21 protein expression was detected at ZT7 (day) than at ZT19 (night) in the Golgi and intracellular vesicles of LECs within mouse skin (Extended Data Fig. 5a,b), indicating diurnal release into the environment. We used quantitative immunofluorescence imaging of non-permeabilized ear whole mounts to resolve the CCL21 gradient in the extracellular interstitial tissue in mice ${ }^{8}$. The use of distance-dependent fluorescence masks (Extended Data Fig. 5c) indicated higher immunoreactivity to CCL21 at ZT7 (day) than at ZT19 (night), particularly in proximity to LVs (Fig. 3d). Elimination of the CCL21 gradient by adding exogenous CCL21, heparinase digestion ${ }^{8}$ or a CCL21 antibody decreased DC migration into LVs at ZT7 compared to control-treated samples and completely abrogated the differences in DC migration between ZT7 (day) and ZT19 (night) (Fig. 3e and Extended Data Fig. 5d-g). $C c r 7^{-/-}$BMDCs exhibited strongly reduced migration into LVs and a lack of rhythmicity compared to BMDCs generated from control mice (Fig. 3f). Synchronized Ccr7 $7^{-/-}$BMDCs at CT24 and CT36 exhibited no differences in migration velocity in collagen gel chemotaxis assays compared to synchronized wild-type (WT) control BMDCs (Fig. 3g), indicating that the circadian expression of CCR7 controlled the rhythmicity of DC migration.

Antibody-mediated blockade of LYVE-1, CD99 and JAM-A in ear explant assays resulted in a marked decrease in $\mathrm{CD}_{11 \mathrm{c}^{+}}$ 


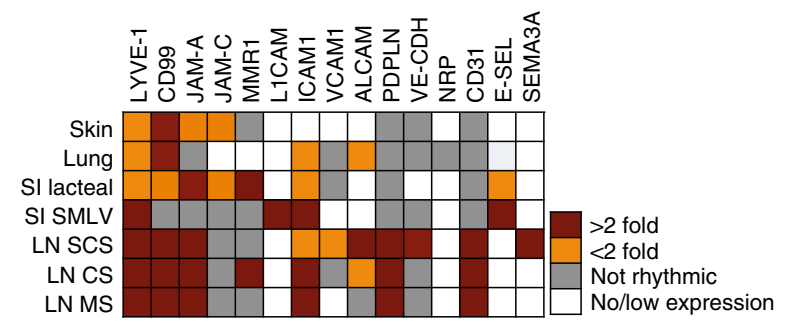

b

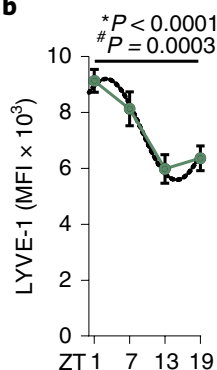

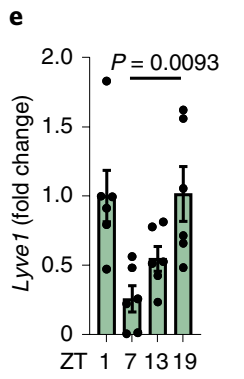

f $\begin{aligned} * & \pm=0.0083 \\ 400 & \stackrel{*}{*} P=0.0032\end{aligned}$

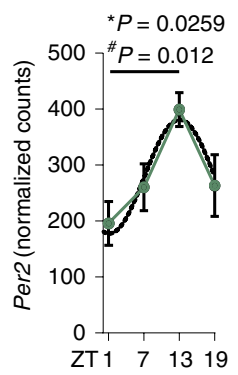

g

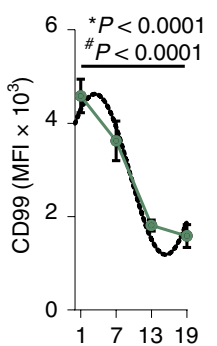

C

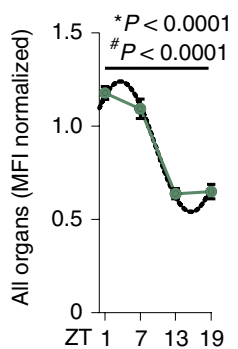

d

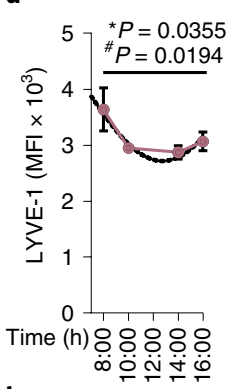

h

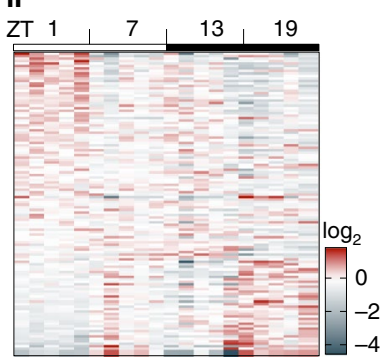

i

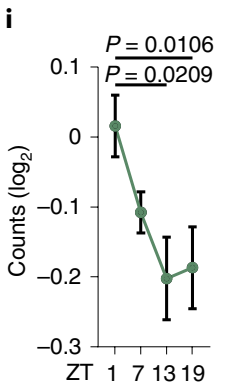

j

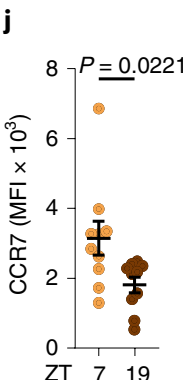

k

Fig. 2 | Diurnal expression of promigratory factors in LECs and DCs. a, Immunofluorescence screen of molecules expressed in LYVE-1+ ear LVs. No/ low expression is $<1.5 \%$ of max mean fluorescence intensity (MFI). Each square represents $n=5$ mice with four ZTs measured. SI, small intestine; SMLV, sub-mucosal lymphatic vessel;SCS, subcapsular sinus;CS, cortical sinus;MS, medullary sinus. b, Expression profiles of molecules in LYVE-1+ ear LVs. c, Integration of all rhythmic expression profiles across organs collapsed into one graph; $n=5$ mice from two independent experiments. $\mathbf{d}$, Expression profile of LYVE-1 in LVs in human skin biopsies from $n=5-9$ individuals; asterisks show results from one-way ANOVA, and number signs show results from cosinor analysis (b-d). e-i, RNA analyses of dermal LECs sorted at four different ZTs. e, Normalized dermal LEC Lyve1 expression; $n=3$ mice representative of two independent experiments; one-way ANOVA with Tukey's post test. f, Normalized clock gene counts determined from RNA sequencing of sorted dermal LECs; $n=5$ mice; asterisks show results from one-way ANOVA, and number signs show results from cosinor analysis. g, Significant Gene Ontology (GO) cluster enrichment between different ZTs of dermal LECs. h, Heat map of significantly rhythmically expressed adhesion genes (GO:0022610) in dermal LECs. i, Integration of all genes from $\mathbf{h}$ collapsed into one graph; $n=82$ genes; one-way ANOVA with Tukey's post test. j, Flow cytometry of CCR7 on

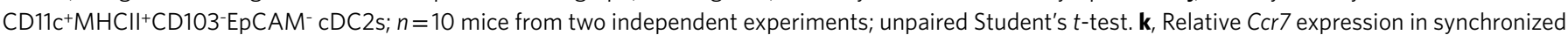
BMDCs across four CTs and normalized to CT18; $n=3$ mice representative of two independent experiments; one-way ANOVA with Tukey's post test. Dotted lines represent fit cosinor curves. All data are represented as mean \pm s.e.m.

DC immigration into skin lymphatics at ZT7 (day) compared to isotype-treated controls, whereas no effects were observed in DC immigration at ZT19 (night) (Fig. 3h and Extended Data Fig. 6a-d). JAM-C blockade ablated diurnal variation but did not reduce DC immigration into afferent lymphatics at ZT7 or ZT19 (Extended Data Fig. 6e), whereas Fc receptor blockade had no effect on DC trafficking into LVs (Extended Data Fig. 6f). The relevance of CD99 in lymphatic migration was confirmed with $C d 99^{-1-}$ mice (Fig. 3i). Because individual blocking approaches abolished the diurnal differences in DC migration and blocked migration into the afferent lymphatics only at ZT7 (day) but not ZT19 (night), we tested whether these signals act redundantly. Combined blockade of LYVE-1, CD99 and JAM-A reduced the migration of DCs into LVs at both ZT7 and ZT19, while additional CCL21 blockade completely inhibited DC infiltration into afferent lymphatics at both time points (Fig. 3j,k and Extended Data Fig. 6g,h). We used distance and distribution analyses to quantify at which step in the rhythmic migration process each molecule was required. Blockade of LYVE-1, CD99 or JAM-A arrested DCs at the level of adhesion to the LEC. By contrast, blocking CCL21 retained the DCs in the interstitial area (Fig. 31 and Extended Data Fig. 6i), indicating that the migration of DCs is tightly controlled by successive interactions of promigratory factors.

Finally, we assessed the role of circadian clock genes in endothelial cells and DCs in controlling DC migration from the skin. DCs from $C d h 5 c r e^{\mathrm{ERT} 2} \mathrm{Bmall}^{\text {flox }}$ mice, in which the circadian transcription factor BMAL1 is deleted in blood endothelial cells and LECs $\left(\right.$ BMAL1 $^{\triangle \mathrm{EC}}$ ), and Prox $1 \mathrm{cre} \mathrm{ERT2}^{\mathrm{ER}}$ mall ${ }^{\text {flox }}$ mice, in which BMAL1 is deleted in LECs (BMAL1 ${ }^{\triangle \mathrm{LEC}}$ ) (Extended Data Fig. 7a,b), showed no rhythmic migration into skin LVs in ears collected at ZT7 and ZT19 compared to Bmall ${ }^{\text {flox }}$ control mice (Fig. 4a,b and Extended Data Fig. 7c,d). In addition, Clec9acreBmall flox mice, which lack BMAL1 in $C D C s\left(B M A L 1^{\triangle C D C}\right)$, showed significantly reduced and non-rhythmic DC migration into LVs in ear skin explants compared 

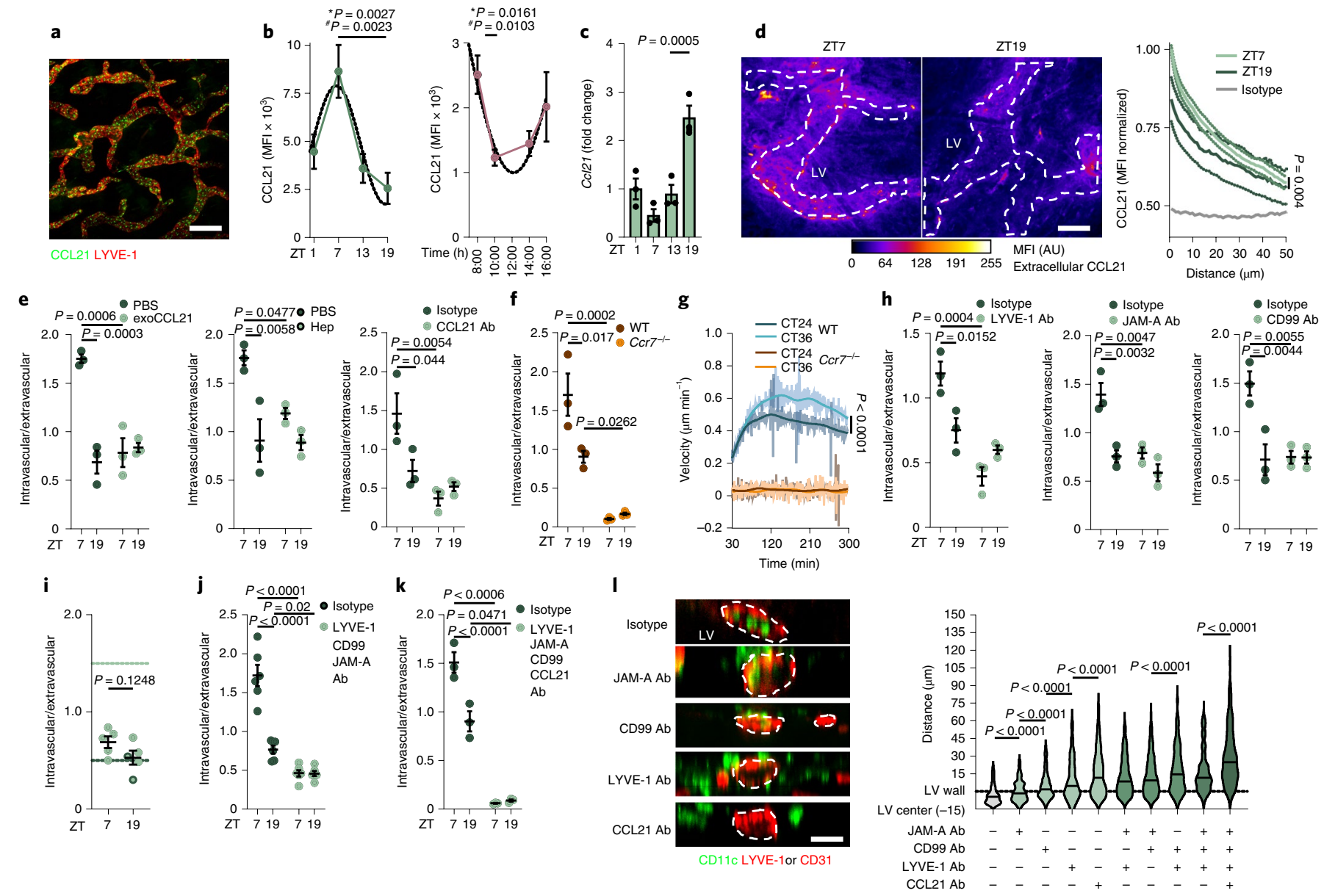

Fig. 3 | Chronopharmacology of circadian DC migration. a, Imaging of CCL21 in permeabilized ears. Image is representative of two independent experiments. Scale bar, $70 \mu \mathrm{m}$. b, Quantitative immunofluorescence screen of CCL21 in LYVE-1+ LVs in mouse (left; $n=5$ mice from two independent experiments) and human (right; $n=5-11$ individuals) skin samples; asterisks show results from one-way ANOVA, and number signs show results from cosinor analysis. c, Normalized CCI21 expression in sorted dermal LECs measured by qPCR. Data are representative of two independent experiments; $n=3$ mice; one-way ANOVA with Tukey's post test. d, Quantitative imaging of non-permeabilized ears for CCL21; $n=5$ mice from two independent experiments; Mann-Whitney U-test. Scale bar, $50 \mu \mathrm{m}$. e, Ear CD11c cells after crawl-in assays and addition of exogenous (exo) CCL21 (left), heparinase (hep) treatment (middle) or anti-CCL21 antibody blockade (right); $n=3$ mice representative of two independent experiments. $\mathbf{f}$, Ear BMDCs after crawl-in assays using Ccr71- or control cells; $n=3$ mice representative of two independent experiments; two-way ANOVA with Sidak post test for $\mathbf{e}$ and $\mathbf{f}$. $g$, Velocity of synchronized WT and $C \mathrm{Cr}^{-1-}$ BMDCs in collagen migration assays; $n=3$ mice representative of two independent experiments; Kruskal-Wallis test with Dunn's post test. h, Ear CD11 $\mathrm{c}^{+}$cells after crawl-in assays and antibody treatment; $n=3$ mice representative of two independent experiments; two-way ANOVA with Sidak post test. i, Ear CD11 $\mathrm{c}^{+}$cells after crawl-in assays using $C d 99^{-/-}$animals (control, dotted lines); $n=5$ mice from two independent experiments; unpaired Student's $t$-test. $\mathbf{j}, \mathbf{k}$, Ear CD11c cells after crawl-in assays and antibody treatments; $n=6$ mice (from two independent experiments for $\mathbf{j}$ ) and $n=3$ mice (representative from two independent experiments for $\mathbf{k}$ ); two-way ANOVA with Sidak post test. I, Quantitative imaging of CD11c ${ }^{+}$cell distance to LVs after ear crawl-in assays; Student's $t$-test; $n=314,451,450,453,374,408,394,396,374$ and 419 cells (left to right) from three mice. Scale bar, $10 \mu m$. Outline represents orthogonal LV view. Dotted lines represent fit cosinor curves. All data are represented as mean \pm s.e.m.

to Bmall ${ }^{\text {flox }}$ control mice (Fig. 4c). Furthermore, synchronized BMDCs generated from Per1 ${ }^{-1-}$ Per2 $^{-/-}$or Bmal1 $^{-1-}$ mice had no rhythmic migration behavior when cells were incubated with ears, in contrast to synchronized WT BMDCs (Fig. 4d,e). These observations indicate that the circadian clock proteins in both LECs and DCs are required for DC rhythmic migration into LVs.

Next, we profiled the expression of promigratory factors in skin LECs by quantitative immunofluorescence. BMAL1 ${ }^{\triangle \mathrm{EC}}$ and BMAL1 ${ }^{\triangle \mathrm{LEC}}$ mice exhibited non-rhythmic LEC expression in CCL21, LYVE-1, CD99, JAM-A and JAM-C, while expression of the non-rhythmically expressed molecule CD31 was not affected (Fig. 4f,g and Extended Data Fig. 8a,b). In addition, the time-of-day differences in the gradient of CCL21 expression were lost in these mice (Fig. 4h,i). cDCs from BMAL1 ${ }^{\Delta \mathrm{CDC}}$ mice did not exhibit rhythmic expression of CCR7, while CCR7 expres- sion in BMAL1 ${ }^{\triangle \mathrm{CDC}}$ LCs was normal compared to LCs collected from Bmal1 ${ }^{\text {flox }}$ control mice (Fig. $4 \mathrm{j}$ and Extended Data Fig. 8c). Distance and distribution imaging analyses indicated that DCs arrested more distant to LECs in the interstitial area of BMAL1 ${ }^{\triangle L E C}$ mice than of BMAL1 ${ }^{\triangle \mathrm{CDC}}$ mice (Fig. $4 \mathrm{k}$ and Extended Data Fig. $8 \mathrm{~d}$ ), indicating that lack of endothelial cell BMAL1 expression resulted in a more marked loss of DC migration into LVs than loss in DC BMAL1. These data and the presence of canonical E-box-binding sites for BMAL1 in the promoter regions of $\mathrm{Ccl} 21$, Ccr7 and Lyve 1 genes (Extended Data Fig. 8e) indicated that these genes are directly regulated by the circadian clock. ChIP assays detected the rhythmic binding of BMAL1 to these promoters, with a peak at ZT13 (Fig. 41 and Extended Data Fig. 8f), indicating that BMAL1 directly controls the signals governing DC migration into the afferent LVs. 

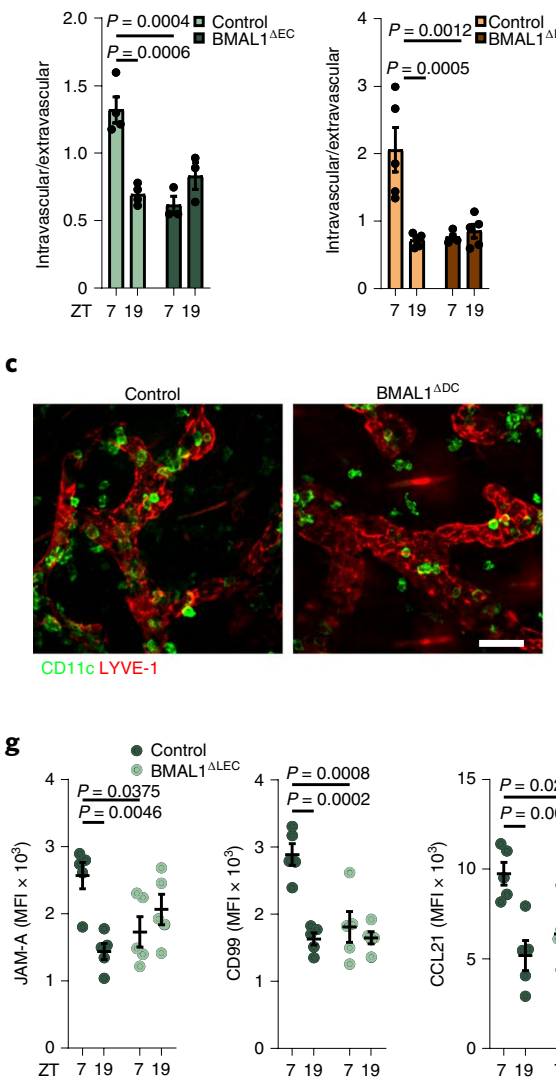

b

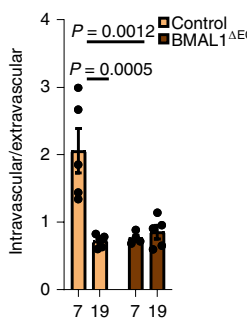

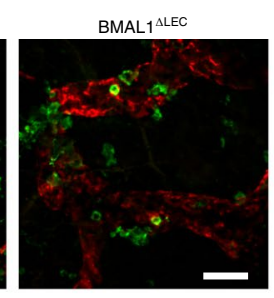
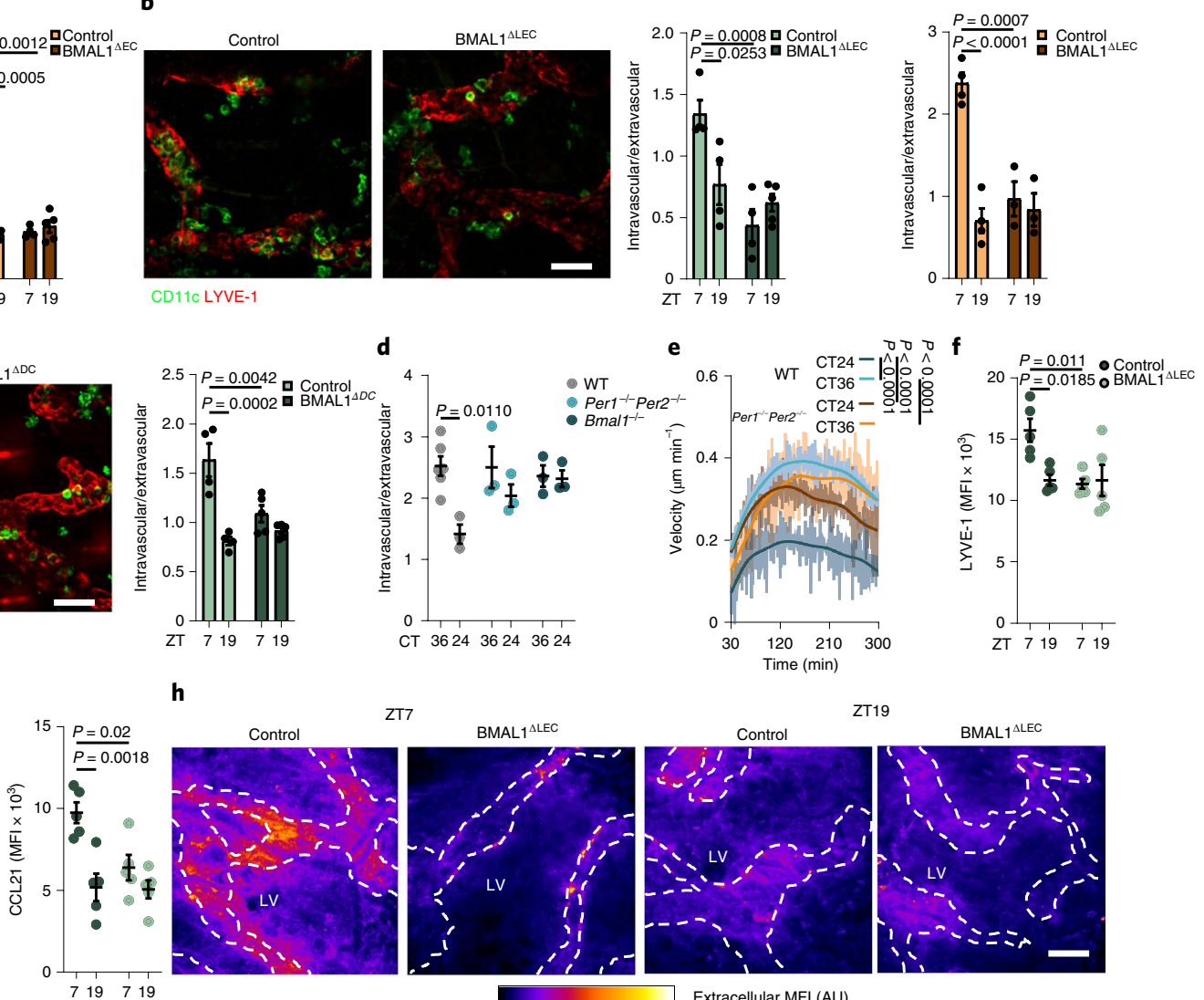

ZT7 BMAL1 ${ }^{\mathrm{ALEC}}$

ZT19

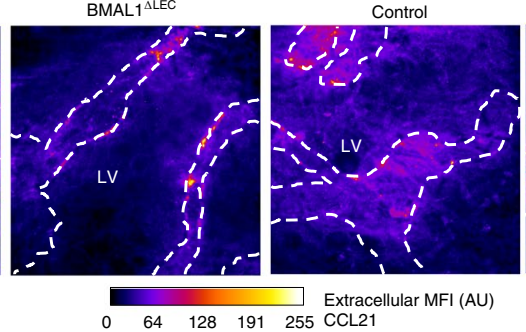

BMAL1 $1^{\triangle L E C}$

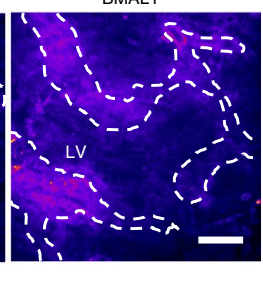

k
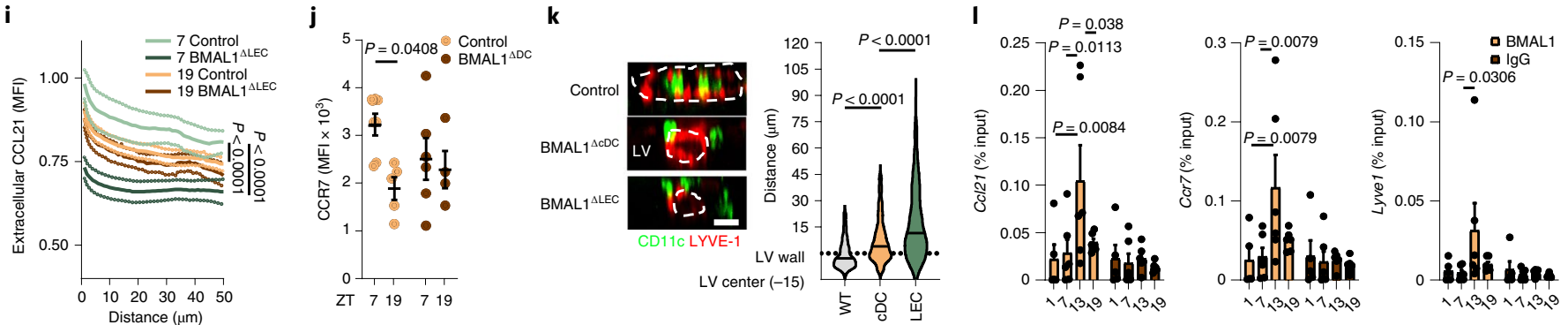

Fig. 4 | Lineage-specific BMAL1 deficiency abrogates rhythms in DC lymphatic trafficking. a-c, Ear CD11c ${ }^{+}$cells (green) or BMDCs (orange) after crawl-in assays with lineage-specific Bmal1-/- animals; $n=4,4,3$ and 3 mice (left) and 5, 5, 4 and 5 mice (right) (a); $n=4,4,4$ and 5 mice (left) and 4, 4, 3 and 3 (right) (b); $n=4,4,5$ and 5 mice (c); data are representative of two independent experiments; two-way ANOVA with Sidak post test. b,c, Representative ear whole-mount images from control and BMAL1 ${ }^{\triangle L E C}(\mathbf{b})$ or BMAL1 ${ }^{\triangle C D C}(\mathbf{c})$ animals. Scale bar, $50 \mu \mathrm{m}$. d, Ear BMDCs after crawl-in assays with synchronized control, Per1 $1-$ Per2 $2^{-/-}$or Bmal1-/- BMDCs; $n=6$ mice for WT cells and $n=3$ for knockout (KO) cells, representative of two independent experiments; two-way ANOVA with Sidak post test. e, Velocity of synchronized WT and Per1 $1-$ Per2 ${ }^{-1-}$ BMDCs in collagen gel migration assays; $n=3$ mice. Data are representative of two independent experiments; Kruskal-Wallis test with Dunn's post test. $\mathbf{f}, \mathbf{g}$, Quantitative immunofluorescence screen of LYVE-1 (f), JAM-A, CD99 and $\mathrm{CCL} 21$ (g) in LYVE-1+ ear LVs; $n=5$ mice from two independent experiments; two-way ANOVA with Sidak post test. h,i, Ear whole-mount imaging (h) and quantification (i) of CCL21 of WT and BMAL1 ${ }^{\triangle \mathrm{LEC}}$ ears; $n=5$ mice from two independent experiments; Kruskal-Wallis test with Dunn's post test. Dashed

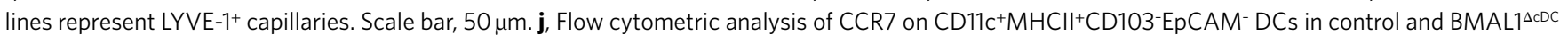
animals; $n=7,5,6$ and 4 mice from two independent experiments; two-way ANOVA with Sidak post test. $\mathbf{k}$, Representative images and quantification of the distance of CD11 $\mathrm{c}^{+}$cells to LVs after ear crawl-in assays; $n=340,431$ and 575 cells from four mice from two independent experiments; unpaired Student's t-test. Scale bar, $10 \mu \mathrm{m}$. Outline represents orthogonal LV view. I, Chromatin immunoprecipitation (ChIP) of BMAL1 binding to promoter regions of CcI21, $\mathrm{C} c r 7$ and Lyve 1 compared to IgG controls; $n=6$ mice from two independent experiments; two-way ANOVA with Sidak post test. All data are represented as mean \pm s.e.m.

In summary, we present here evidence that the migration of DCs into skin lymphatics is a rhythmic process. We found that CCL21, CCR7 and LYVE-1 are under the direct control of the circadian clock gene BMAL1, implicating the clock as an essential and broad regulator of DC migration. As this process is fundamental for the generation of adaptive immunity, it should prove useful to exploit in vaccination and immunotherapeutic regimens, considering that rhythmicity in innate and adaptive immune responses is maintained in inflammatory reactions $s^{13,14,21,25-27}$. We also observed analogous but phase-shifted rhythmicity in the expression of human promigratory factors, suggesting that these functional diurnal differences observed in mice should also apply to humans. 


\section{Online content}

Any methods, additional references, Nature Research reporting summaries, source data, extended data, supplementary information, acknowledgements, peer review information; details of author contributions and competing interests; and statements of data and code availability are available at https://doi.org/10.1038/ s41590-021-01040-x.

Received: 11 May 2021; Accepted: 31 August 2021; Published online: 18 October 2021

\section{References}

1. Forster, R. et al. CCR7 coordinates the primary immune response by establishing functional microenvironments in secondary lymphoid organs. Cell 99, 23-33 (1999).

2. Ulvmar, M. H. et al. The atypical chemokine receptor CCRL1 shapes functional CCL21 gradients in lymph nodes. Nat. Immunol. 15, 623-630 (2014).

3. Palomino-Segura, M. \& Hidalgo, A. Circadian immune circuits. J. Exp. Med. 218, e20200798 (2021).

4. Scheiermann, C., Gibbs, J., Ince, L. \& Loudon, A. Clocking in to immunity. Nat. Rev. Immunol. 18, 423-437 (2018).

5. Ohl, L. et al. CCR7 governs skin dendritic cell migration under inflammatory and steady-state conditions. Immunity 21, 279-288 (2004).

6. Saeki, H., Moore, A. M., Brown, M. J. \& Hwang, S. T. Cutting edge: secondary lymphoid-tissue chemokine (SLC) and CC chemokine receptor 7 (CCR7) participate in the emigration pathway of mature dendritic cells from the skin to regional lymph nodes. J. Immunol. 162, 2472-2475 (1999).

7. Johnson, L. A. \& Jackson, D. G. Inflammation-induced secretion of CCL21 in lymphatic endothelium is a key regulator of integrin-mediated dendritic cell transmigration. Int. Immunol. 22, 839-849 (2010).

8. Weber, M. et al. Interstitial dendritic cell guidance by haptotactic chemokine gradients. Science 339, 328-332 (2013).

9. Russo, E. et al. Intralymphatic CCL21 promotes tissue egress of dendritic cells through afferent lymphatic vessels. Cell Rep. 14, 1723-1734 (2016).

10. Johnson, L. A. et al. Dendritic cells enter lymph vessels by hyaluronan-mediated docking to the endothelial receptor LYVE-1. Nat. Immunol. 18, 762-770 (2017).

11. Torzicky, M. et al. Platelet endothelial cell adhesion molecule-1 (PECAM-1/ CD31) and CD99 are critical in lymphatic transmigration of human dendritic cells. J. Invest. Dermatol. 132, 1149-1157 (2012).

12. Cera, M. R. et al. Increased DC trafficking to lymph nodes and contact hypersensitivity in junctional adhesion molecule-A-deficient mice. J. Clin. Invest. 114, 729-738 (2004).

13. Druzd, D. et al. Lymphocyte circadian clocks control lymph node trafficking and adaptive immune responses. Immunity 46, 120-132 (2017).
14. Suzuki, K., Hayano, Y., Nakai, A., Furuta, F. \& Noda, M. Adrenergic control of the adaptive immune response by diurnal lymphocyte recirculation through lymph nodes. J. Exp. Med. 213, 2567-2574 (2016).

15. Scheiermann, C., Kunisaki, Y. \& Frenette, P. S. Circadian control of the immune system. Nat. Rev. Immunol. 13, 190-198 (2013).

16. Shimba, A. et al. Glucocorticoids drive diurnal oscillations in $\mathrm{T}$ cell distribution and responses by inducing interleukin-7 receptor and CXCR4. Immunity 48, 286-298 (2018).

17. Pflicke, H. \& Sixt, M. Preformed portals facilitate dendritic cell entry into afferent lymphatic vessels. J. Exp. Med. 206, 2925-2935 (2009).

18. Balsalobre, A., Damiola, F. \& Schibler, U. A serum shock induces circadian gene expression in mammalian tissue culture cells. Cell 93, 929-937 (1998).

19. Yoo, S. H. et al. PERIOD2::LUCIFERASE real-time reporting of circadian dynamics reveals persistent circadian oscillations in mouse peripheral tissues. Proc. Natl Acad. Sci. USA 101, 5339-5346 (2004).

20. Nobis, C. C. et al. The circadian clock of CD8 T cells modulates their early response to vaccination and the rhythmicity of related signaling pathways. Proc. Natl Acad. Sci. USA 116, 20077-20086 (2019).

21. Hopwood, T. W. et al. The circadian regulator BMAL1 programmes responses to parasitic worm infection via a dendritic cell clock. Sci. Rep. 8, 3782 (2018).

22. Early, J. O. et al. Circadian clock protein BMAL1 regulates IL-1 $\beta$ in macrophages via NRF2. Proc. Natl Acad. Sci. USA 115, E8460-E8468 (2018).

23. Jackson, D. G. Leucocyte trafficking via the lymphatic vasculaturemechanisms and consequences. Front. Immunol. 10, 471 (2019).

24. Mauvoisin, D. et al. Circadian clock-dependent and -independent rhythmic proteomes implement distinct diurnal functions in mouse liver. Proc. Natl Acad. Sci. USA 111, 167-172 (2014).

25. Gibbs, J. et al. An epithelial circadian clock controls pulmonary inflammation and glucocorticoid action. Nat. Med. 20, 919-926 (2014).

26. He, W. et al. Circadian expression of migratory factors establishes lineage-specific signatures that guide the homing of leukocyte subsets to tissues. Immunity 49, 1175-1190 (2018).

27. Sutton, C. E. et al. Loss of the molecular clock in myeloid cells exacerbates T cell-mediated CNS autoimmune disease. Nat. Commun. 8, 1923 (2017).

Publisher's note Springer Nature remains neutral with regard to jurisdictional claims in published maps and institutional affiliations.

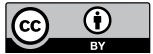

Open Access This article is licensed under a Creative Commons

Attribution 4.0 International License, which permits use, sharing, adaptation, distribution and reproduction in any medium or format, as long as you give appropriate credit to the original author(s) and the source, provide a link to the Creative Commons license, and indicate if changes were made. The images or other third party material in this article are included in the article's Creative Commons license, unless indicated otherwise in a credit line to the material. If material is not included in the article's Creative Commons license and your intended use is not permitted by statutory regulation or exceeds the permitted use, you will need to obtain permission directly from the copyright holder. To view a copy of this license, visit http://creativecommons. org/licenses/by/4.0/.

(c) The Author(s) 2021 


\section{Methods}

Mouse models. Male WT C57BL/6N mice aged 6-8 weeks were purchased from either Charles River Laboratories or Janvier labs. Cdh5-creERT2 (B6) mice were obtained as a gift from R. Adams (Max Planck Institute for Molecular Biomedicine) and crossbred with Bmal1 ${ }^{\text {flox/flox }}$ (B6) mice obtained from Jackson Laboratories to target blood endothelial cells and LECs. Prox1-creERT2 (B6) mice (Jackson Laboratories) were crossbred with Cdh5-creERT2-Bmal1 ${ }^{\text {flox/llox }}$ mice to obtain Prox1-creERT2-Bmall flox/flox mice to specifically target LECs. Cdh5-creERT2 and Prox1-creERT2 mice were given intraperitoneal tamoxifen injections for five consecutive days at the age of 6 weeks to induce Cre recombinase expression and excise Bmal1 at the respective flox regions. EYFP; Clec9acre mice were crossbred with $B$ mall $1^{\text {flox/flox }}$ mice to target $\mathrm{CDCs}^{28}$. Cd99 $9^{-/-}$mice and bone marrow (BM) cells from $\mathrm{Ccr}^{-1-}$ mice, $\mathrm{Bmall}^{-1-}$ mice and Per2::Luc mice aged 6-8 weeks were used. Primary BM cells from Per $^{-/-}$Per $2^{-/-}$mice were provided by J. Ripperger and U. Albrecht (University of Fribourg)

All animals were housed under a 12-h light/12-h dark schedule with ad libitum access to water and food in the Core Facility Animal Models at the Biomedical Centre (Ludwig-Maximilians-Universität) or the University of Geneva. To facilitate simultaneous experiments at different time points of the day, light cabinets (Techniplast) were used to shift animals to different light-dark cycles. For experiments requiring altered lighting patterns, mice were either housed in complete darkness or given 2 weeks to adapt to inverted lighting patterns, respectively. All animal procedures and experiments were in accordance with the ministry of animal welfare of the region of Oberbayern and with the German law of animal welfare or were approved and performed in accordance with the guidelines of the animal research committee of Geneva.

Human skin biopsy sampling. Skin biopsies were taken from male and female adults (mean age, 74 years) presenting at the dermatosurgery unit of the Geneva University Hospitals for the removal of skin tumors under local anesthesia. Written informed consent was obtained from each individual. Sampling was conducted according to the Declaration of Helsinki and approved by the Commission Cantonale d'Ethique de la Recherche of the University Hospitals of Geneva. Samples were taken from excessive, tumor-free surrounding skin known as 'Burow's triangles' during the reconstruction of dermatosurgical excisions. No extra incisions were made to obtain the sample, and the size of the original tumor excision was not altered by this study. The sample was placed into normal saline solution immediately after it had been excised, and the time of day was noted. Samples were then embedded in optimal cutting temperature compound (OCT) and shock frozen until sectioning.

BMDC cell culture, synchronization and labeling. BMDCs used for exogenous crawl-in assays were cultured using R10 medium (RPMI, 10\% fetal calf serum (Gibco), $2 \mathrm{mM}$ L-glutamine, $1 \%$ penicillin/streptomycin and $50 \mu \mathrm{M}$ $\beta$-mercaptoethanol) supplemented with $20 \mathrm{ng} \mathrm{ml}^{-1}$ granulocyte-macrophage colony-stimulating factor (GM-CSF) hybridoma supernatant. GM-CSF-producing hybridoma cells were used as described ${ }^{29}$. GM-CSF concentration in the supernatant was measured using an mGM-CSF Quantikine ELISA kit (R\&D Systems).

Cells were cultured for $10 \mathrm{~d}$. At day 10, all non-adherent cells were collected and immersed in R10 medium supplemented with $10 \mathrm{ng} \mathrm{ml}^{-1} \mathrm{GM}-\mathrm{CSF}$ and stimulated with $100 \mathrm{ng} \mathrm{ml}^{-1}$ LPS (Sigma-Aldrich) for $24 \mathrm{~h}$. Viability and purity of BMDCs were checked by analysis of MHCII and CD11c expression on DAPI-negative BMDCs.

To synchronize BMDCs, on day $8,7 \times 10^{6}$ BMDCs per sample culture were replated. The next day, BMDCs were placed in 50\% horse serum (Merck, H1138) for $2 \mathrm{~h}$ (shock) $)^{20,22}$ with two subsequent washes and on day 10 received the LPS challenge.

After maturation and stimulation, the required number of cells was incubated with $5 \mu \mathrm{M}$ CellTrace Violet or CellTrace Yellow (1:1000; Thermo Fisher), thoroughly washed and adjusted to a final concentration of $5-7.5 \times 10^{5}$ cells per $\mathrm{ml}$ to be used for migration assays.

Luciferase-expressing BMDCs. For BMDC synchronization experiments, BM was collected from Per2::Luc mice and grown as described above. On day 8 , all non-adherent cells were collected and resuspended at $2 \times 10^{5}$ cells per $\mathrm{ml}$ in complete medium. Cells were replated into $35-\mathrm{mm}$ dishes $(2 \mathrm{ml}$ per dish) and left overnight to recover. To synchronize, cells were incubated in complete medium with $50 \%$ horse serum for $2 \mathrm{~h}$. Following synchronization, cells were washed gently with complete medium to remove excess serum and left in $2 \mathrm{ml}$ of fresh medium containing $100 \mu \mathrm{M}$ luciferin (BioLume) and LPS. Dishes were sealed with parafilm and transferred to a LumiCycle (Actimetrics) for bioluminescence recording. Photon counts per minute were detrended using a $24-\mathrm{h}$ moving average to calculate relative bioluminescence.

Exogenous BMDC crawl-in migration assay. For exogenous BMDC crawl-in migration assays, mice were killed according to animal protocols. Protocols were adapted ${ }^{17}$, ears were carefully collected and their dorsal and ventral sides were separated and quickly rinsed in medium. Ears were placed in custom-built imaging chambers and immobilized. Labeled BMDCs $(25,000-37,500)$ were added onto ears for 10-15 min. After washing off any unbound BMDCs, ears were completely covered with R10 medium and incubated for a maximum of $3 \mathrm{~h}$. Ear whole mounts were stained with different combinations of LYVE-1, CD31 or podoplanin and were subsequently fixed in $4 \%$ paraformaldehyde. Images of ears were obtained as whole mounts using a Zeiss Axio Examiner.Z1 confocal spinning disk microscope equipped with 405-, 488-, 561- and 640-nm laser sources.

For end-point analysis, at least eight images per ear whole mount were taken from LVs (precollecting and capillaries). The number of cells outside or inside the vessels was counted using three-dimensional (3D) visualization tools in Fiji and Slidebook 6.0 as well as orthogonal views and optical slicing. This number was then normalized to the calculated volume of the vessels.

For short crawl-ins, distance-dependent zone segmentation of the interstitial area and accumulation analyses were used. After BMDC addition and wash-off, ears were incubated in medium for 0-60 min, stained and imaged as described above. In each $z$ projection, CD $31^{+}$LVs were manually outlined, a binary mask was generated and LV distance-dependent maps were applied to create specific segments in the interstitial area using a custom-made script in Matlab. BMDCs were manually counted within these segments, and the relative distribution was calculated.

Endogenous skin DC mobilization and crawl-in assay. For endogenous leukocyte crawl-in migration assays, mice were killed according to animal protocols, and collected ears were split. After an initial wash, ear halves were stored on medium with the dermis facing down for $6 \mathrm{~h}$ or $24 \mathrm{~h}$. Afterwards, ear sheets were fixed in $4 \%$ paraformaldehyde and stained for CD11c, LYVE-1 and/or CD31. Cells were counted and normalized, and the in-versus-out ratio was generated as described above.

Live imaging of BMDC crawl-in assays. For live imaging of exogenous crawl-in assays, ear whole mounts were prepared as described above; however, they were subjected to an initial staining before being used for the trafficking assay. Ears were stained for laminin with a primary antibody and then probed with a secondary FITC-conjugated antibody in medium at room temperature before receiving $50,000-75,000$ BMDCs for $10 \mathrm{~min}$. Unbound BMDCs were washed off, and the ear half was immersed in phenol red-free R10 medium supplemented with $10 \mathrm{mM}$ HEPES (Sigma). Imaging was performed in a $5 \% \mathrm{CO}_{2}$ chamber at $37^{\circ} \mathrm{C}$ with one $30-40-\mu \mathrm{m}$-deep 3D image acquired every $100 \mathrm{~s}$ for $45-60 \mathrm{~min}$. Migration paths, distance plots and velocity, directionality, Euclidian and accumulated distances were calculated using the TrackMate and manual tracking plugins in Fiji together with the Ibidi chemotaxis and migration tool.

Functional blocking of proteins and abrogation of chemokine gradients. To investigate the functional relevance of proteins involved in rhythmic dermal DC trafficking, antibodies or enzymes were used. Ears of exogenous crawl-in assays were incubated with medium containing diluted neutralization antibodies before the migration assay for $1 \mathrm{~h}$ at room temperature or directly added to the endogenous crawl-in medium for $24 \mathrm{~h}$ (see antibody list in the Supplemental Information).

For abrogating the extracellular CCL21 gradient, ears were incubated with either heparinase II and IV ( $100 \mathrm{mIU}$; Sigma) at $37^{\circ} \mathrm{C}$ for $1 \mathrm{~h}$ or placed in PBS containing $0.1 \%$ bovine serum albumin and $0.6 \mu \mathrm{g} \mathrm{ml}^{-1} \mathrm{CCL} 21$ (Peprotech) at $4{ }^{\circ} \mathrm{C}$ for $90 \mathrm{~min}$ before the migration. Unbound CCL21 or remaining heparinase was washed off afterwards.

Lymphatic distance analysis. To measure the distance between DCs and the LV, a combination of orthogonal views, maximum $z$ projections and 3D viewers was used. At least $250 \mathrm{CD} 11 \mathrm{c}^{+}$cells per biological replicate were taken for analysis. To calculate the average diameter of LVs, 40 vessels were used as a proxy. The distance between LV and DC center was then manually measured using Fiji.

Crawl-out assay. Collected and split ears were washed for $2 \mathrm{~h}$ and stored in R10 medium for $24 \mathrm{~h}$ supplemented with $1 \mu \mathrm{g} \mathrm{ml}^{-1} \mathrm{CCL} 21$. The next day, ears were collected and gently digested for $20 \mathrm{~min}$ at $37^{\circ} \mathrm{C}$ with collagenase IV $\left(1 \mathrm{mg} \mathrm{ml}^{-1}\right.$; C5138, Sigma), DNase (0.2 $\mathrm{mg} \mathrm{ml}^{-1}$; Roche) and dispase II (0.2 $\mathrm{mg} \mathrm{ml}^{-1}$; Sigma). After digestion, cells were filtered through a $70-\mu \mathrm{m}$ cell strainer, washed and resuspended in PBS supplemented with $2 \%$ fetal calf serum and 2 mM EDTA (Sigma). Simultaneously, the medium containing the emigrated DCs was collected, and both the ear DC and medium DC populations were first Fc receptor blocked with anti-mouse CD16/CD32 for $5 \mathrm{~min}$ at room temperature and subsequently stained with fluorescence-conjugated antibodies for $30 \mathrm{~min}$ at $4^{\circ} \mathrm{C}$. For CCR7, the stain was performed separately at $37^{\circ} \mathrm{C}$ before the other staining step. DAPI and full-bright counting beads (Thermo Fisher) were added to cells, which were then analyzed by flow cytometry using a Gallios flow cytometer (Beckman Coulter) equipped with 405-, 488- and 633-nm lasers or a BD Fortessa flow cytometer (405-, 488-, 561- and 633-nm lasers; BD Biosciences).

In vitro DC migration assay. Synchronized BMDCs $(500,000)$ were washed and used for in vitro chemotaxis migration assays as described ${ }^{30,31}$. For preparation of 
the collagen gel mixture, $30 \mu \mathrm{l}$ of $10 \times \mathrm{MEM}$ and $15 \mu \mathrm{l}$ of $7.5 \%$ sodium bicarbonate were mixed until the solution turned pink. Next, $225 \mu$ l of Nutragen (purified bovine collagen I solution $6 \mathrm{mg} \mathrm{ml}^{-1}$ ) was added and mixed homogenously. Two hundred microliters of this collagen gel mixture and $100 \mu \mathrm{l}$ of the cell suspension were mixed carefully to avoid the formation of air bubbles. Two hundred and eighty microliters of the mixture was pipetted into custom-made chambers. After polymerization for $75 \mathrm{~min}$ at $37^{\circ} \mathrm{C}$ and $5 \% \mathrm{CO}_{2}$, gels were overlaid with $80 \mu \mathrm{l}$ of CCL21 $\left(1 \mu \mathrm{ml}^{-1}\right)$. Afterwards, the chambers were sealed with paraffin.

For imaging of cell migration, one picture per condition was recorded every $60 \mathrm{~s}$ for $5 \mathrm{~h}$ with an inverted wide-field Leica DMi8 microscope using a $\times 4$ objective, a Leica Lumencor SpectraX multi-LED light source, an incubation chamber set to $37^{\circ} \mathrm{C}$ and a heated stage.

For cell migration analysis, the first 30 frames of the recordings were excluded due to initial image instability caused by technical reasons. Frames 31 to 300 were analyzed in ImageJ using a custom-made script ${ }^{32}$.

Topical FITC ear stimulation. FITC ( $8 \mathrm{mg} \mathrm{ml}^{-1}$; Sigma) in 1:1 dibutyl phthalate (Sigma) and acetone (Sigma) was thoroughly vortexed before use. Mice were anesthetized by isoflurane inhalation, and $25 \mu \mathrm{l}$ of this solution was applied to the ear skin and left for $24 \mathrm{~h}$, after which ears were collected for imaging analyses.

Immunofluorescence staining. To measure the expression level of adhesion molecules in LECs, mouse organs (skin, inguinal LN, lung and small intestine) were collected at four time points, placed in OCT (TissueTec), shock frozen on dry ice and stored at $-80^{\circ} \mathrm{C}$. The next day, organs were thawed to $-20^{\circ} \mathrm{C}$ and sectioned at a thickness of $10 \mu \mathrm{m}$ on a cryostat (Leica) ${ }^{26}$. Tissue sections were stored at $-80^{\circ} \mathrm{C}$ or were directly thawed to room temperature and encircled with a hydrophobic alcohol-resistant pen. Sections were fixed with cold methanol (100\%; Sigma) for 10 min, washed, blocked and permeabilized in PBS containing Triton X-100 $(0.5 \%$; Sigma) and normal goat serum (20\%; Sigma). Sections were always stained for LYVE-1 in combination with the proteins of interest (see antibody list in the Supplementary Information) overnight at $4^{\circ} \mathrm{C}$. The next day, samples were washed with PBS and imaged.

To stain CCL21 on tissue slides, the blocking step was expanded using avidin (20\%). Slides were thoroughly washed and incubated in PBS containing $20 \%$ biotin (Vector Labs), and sections were stained for LYVE-1 and CCL21-biotin overnight at $4{ }^{\circ} \mathrm{C}$. The next day, samples were washed with PBS and incubated in labeled streptavidin $(2.5 \mu \mathrm{g}$ per slice $)$ at room temperature for $15 \mathrm{~min}$.

For quantification, five images of different regions of one section from one mouse were acquired, averaged and counted as one biological replicate. The average MFI of five isotype stainings was deducted from the MFI of the protein staining of interest. The MFI was then normalized to the second highest value of all ZTs. Fold change values of MFI between ZTs were generated and shown as a heat map.

Whole-mount immunofluorescence staining. To better understand the 3D architecture of proteins expressed on or in LECs, ears were whole mounted and 3D imaged. Fixation of ears was performed before or after staining with antibodies. In the case of staining for $\mathrm{CD} 31$ or PROX-1, fixation was done after staining. If staining for LYVE-1, podoplanin, laminin, CCL21, GOLPH4, VE-cadherin or $\mathrm{CD} 11 \mathrm{c}$, ears were fixed before staining.

For staining of GOLPH4 and CCL21, ears were washed the next day and stained using secondary antibodies for $2 \mathrm{~h}$ at room temperature. Imaging of whole mounts was performed as described above.

CCL21 gradient analysis. To visualize and analyze the extracellular CCL21 gradient, collected and fixed ears were carefully washed and blocked in R10 medium containing avidin $(20 \%)$ for $1 \mathrm{~h}$ as previously described ${ }^{8}$. After leaving the ears in PBS and biotin (20\%) for $30 \mathrm{~min}$, they were stained for LYVE-1 and CCL21 (Biotin) overnight at $4^{\circ} \mathrm{C}$. The next day, ears were incubated in PE-labeled streptavidin for $3 \mathrm{~h}$ at $4^{\circ} \mathrm{C}$ in the dark and imaged.

Intensities of interstitial CCL21 chemokine signals were quantified on maximum intensity projections ranging between 30 - and $40-\mu \mathrm{m} z$ stacks with a $z$ step size of $1 \mu \mathrm{m}$. Using custom-written Matlab scripts, LV capillaries were manually outlined by an experimentor blinded to the respective experimental condition and converted into binary masks. Next, a Euclidean distance transform was computed on each binary mask, resulting in the shortest Euclidean distance of each non-masked pixel to its nearest mask border/LV. Euclidean distances were rounded and translated into metric units via the microscope's embedded pixel resolution. The distance masks were then applied to their respective CCL21 stainings, and fluorescent intensities were extracted and averaged across distances, resulting in a distance-dependent fluorescent intensity metric per image. Average intensity images of CCL21 staining were integrated into distance-dependent fluorescence intensities. Five average distance-dependent fluorescence intensities from different locations within one mouse ear were averaged as one biological replicate. All samples from ZT7 and ZT19 were normalized to the highest average fluorescence intensity of ZT7. Isotype stainings were taken as negative controls.
CCL21 localization analysis. To localize CCL21 intracellularly, ears were fixed, permeabilized and blocked as mentioned above. After staining for CCL21, LYVE-1 and GOLPH4, using primary and appropriate secondary antibodies, at least five different regions per ear were imaged. Thirty- to forty-micron $z$ stacks with a step size of $1 \mu \mathrm{m}$ were $z$ projected and analyzed using Fiji. CCL21 signal intensity was quantified in LYVE- $1^{+}$cells in either GOLPH $4^{\text {high }}$ or GOLPH $4^{\text {low }}$ regions. The MFI of isotype controls was then deducted from the CCL21 staining.

Sorting of LECs. To sort dermal LECs for RNA sequencing, four ears from two mice were pooled per biological replicate and time point. Dermal cells were isolated as stated above and prepared for sorting on a FACSAria IIIu (BD) equipped with four lasers $(405,488,561$ and $633 \mathrm{~nm}$ ) in the Core Facility Flow Cytometry at Biomedical Centre Munich. Live podoplanin ${ }^{+} \mathrm{CD} 31^{+}$LECs were sorted directly into either $350 \mu \mathrm{l}$ of TriZol-LS (Thermo Fisher in the case of RNA sequencing) or $350 \mu \mathrm{l}$ of RLT buffer with $\beta$-mercaptoethanol (1:100) (Qiagen and Sigma, respectively, in the case of qPCR) at $4^{\circ} \mathrm{C}$ using a $100-\mu \mathrm{m}$ nozzle and a purity of $>90 \%$ as determined by purity checks after every sort. Cell numbers sorted ranged between 3,000 and 5,000 cells per two pooled ear pairs. Directly after sorting, samples were shock frozen on dry ice and stored for further analysis.

RNA sequencing. RNA of sorted dermal LECs collected in TriZol-LS was purified using a Direct-zol RNA mini prep kit (ZymoResearch) following the manufacturer's instructions. Isolated RNA was quantified using a Nanodrop (Thermo Fisher) and analyzed on a Bioanalyzer (Agilent) using an RNA 6000 Nano or Pico kit (Agilent). Eluted total RNA (75 ng) was digested with DNase (Thermo Fisher) to remove DNA contamination. An additional purification step with RNAClean XP beads (Agencourt) was performed. The purified, bead-bound RNA was directly used as input in the SMART-Seq v4 Ultra Low Input RNA kit (TaKaRA Bio). Full-length cDNA was generated following the manufacturer's instructions. Full-length cDNA was quantified using a Qubit dsDNA HS Assay kit (Invitrogen) with a Qubit fluorometer. Finally, sequencing libraries were generated using $500 \mathrm{pg}$ of full-length cDNA following the NexteraXT protocol (Illumina). Libraries were quantified on a Bioanalyzer (Agilent) using a DNA $1000 \mathrm{kit}$ (Agilent) and sequenced on a HiSeq1500 system (Illumina) with a read length of 100 nucleotides in single-end mode.

For data processing, obtained transcriptome profiles were processed on a Galaxy web interface hosted by LAFUGA (Gene Center). After demultiplexing and trimming, data were mapped against the mouse genome (mm10) using RNA-STAR mapper (Galaxy version 2.5.2b-0). Abundant reads were counted using HTSeq-count (Galaxy version 1.0.0). Afterwards, gene expression analysis to detect differentially expressed genes was performed using DESeq2 (Galaxy version 2.11.40.6), setting the false discovery rate to $<0.05$. The sequencing data have been deposited in the NCBI Gene Expression Omnibus (GEO) and are accessible through GEO series accession number GSE184758.

GO cluster enrichment analysis. GO cluster enrichments were generated using metascape.org (ref. ${ }^{33}$ ). Enriched genes from the DESeq2 analysis with $P \leq 0.05$ were organized in gene lists and screened for GO biological process enrichment. A $P$ value of $\leq 0.01$, a minimum count of 3 and an enrichment factor of $\geq 1.5$ were used as cutoff parameters. Genetic terms were collected and grouped into clusters based on their membership similarities.

To compare the biological adhesion (GO:0022610) gene counts, sequencing results were filtered for GO:0022610 cluster genes, and significantly up- and downregulated genes were compiled in a heat map.

qPCR. RLT-sorted LECs or lysed synchronized BMDCs were thawed and thoroughly mixed. RNA was isolated using the RNeasy micro kit (Qiagen) according to the manufacturer's instructions. RNA samples were analyzed using a NanoDrop2000 (Thermo Scientific) to determine RNA concentration and quality. Obtained RNA was directly reverse transcribed into cDNA with the High Capacity cDNA Reverse Transcription kit (Applied Biosystems). cDNA samples were stored at $-20^{\circ} \mathrm{C}$ before use. $\mathrm{qPCR}$ was performed with a StepOnePlus Real-Time PCR System (Applied Biosystems) in 96-well plates with SYBR green-compatible primers. Each qPCR sample was analyzed in duplicate. The total reaction volume was $10 \mu \mathrm{l}$ and contained $5 \mu \mathrm{l}$ of SYBR green, $1 \mu \mathrm{l}$ of primer mix $(5 \mu \mathrm{M}), 3 \mu \mathrm{l}$ of water and $1 \mu \mathrm{l}$ of cDNA. Gene expression levels were normalized to the housekeeping gene Rpl32.

ChIP analyses. After animals were killed, draining LNs were snap frozen on dry ice and stored at $-80^{\circ} \mathrm{C}$ before processing. Tissue was dounce homogenized in $1 \mathrm{ml}$ of homogenization buffer (10 mM HEPES-KOH, $10 \mathrm{mM} \mathrm{KCl}, 5 \mathrm{mM} \mathrm{MgCl}_{2}, 0.5 \mathrm{mM}$ DTT and $1 \times$ Complete Protease Inhibitor (Roche)). The homogenate was fixed in PBS containing $1 \%$ final formaldehyde (Thermo Fisher Scientific). Nuclei were isolated, and chromatin suspensions were obtained through sonication (Diagenode Bioruptor) to obtain fragments of $0.2-0.8$ kilobases in size. Immunoprecipitation was performed with anti-BMAL1 (D2L7G, Cell Signaling Technology) or control IgG (Abcam). DNA was isolated with MinElute PCR Purification kits (Qiagen). DNA concentration was determined by using a Qubit dsDNA HS kit (Thermo Fisher Scientific). Real-time ChIP-qPCR was performed by use of SYBR Green 
Master Mix (Roche) in a LightCycler 480 II (Roche). Occupancy of BMAL1 at the Ccr7, Ccl21, Lyve1 and Per2 promoters was quantified by qPCR targeting regions identified as containing E-boxes using the SCOPE motif finder and EPFL eukaryotic database. Relative enrichment was determined as percentage of the input.

Statistics. All figures represent merged data from independent biological replicates, and exceptions are explained in the figure legends. Adequate control experiments were individually performed for each biological replicate. Validation of replicates was performed independently, and they were pooled only when all replicates demonstrated invariable results. Complete statistical analyses, including the cosinor test, were done using GraphPad Prism, taking into consideration normal and non-normal data distributions; ${ }^{*}$ or ${ }^{\#}, P<0.05 ;{ }^{* *}$ or ${ }^{* \#}, P<0.01$; ${ }^{* * *}$ or ${ }^{\# \# *}$ $P<0.001 ; * *+* *$ or ${ }^{\# \# \#,}, P<0.0001$.

Reporting Summary. Further information on research design is available in the Nature Research Reporting Summary linked to this article.

\section{Data availability}

All data that support the conclusions of this paper are available at https://doi. org/10.26037/yareta:alphbk7uinejtgflybg7utlasq. Source data are provided with this paper. The sequencing data have been deposited in the NCBI GEO and are accessible through GEO series accession number GSE184758.

\section{Code availability}

All adapted scripts published in previous papers are available from the corresponding author on request.

\section{References}

28. Schraml, B. U. et al. Genetic tracing via DNGR-1 expression history defines dendritic cells as a hematopoietic lineage. Cell 154, 843-858 (2013).

29. Zal, T., Volkmann, A. \& Stockinger, B. Mechanisms of tolerance induction in major histocompatibility complex class II-restricted T cells specific for a blood-borne self-antigen. J. Exp. Med. 180, 2089-2099 (1994).

30. Renkawitz, J. et al. Nuclear positioning facilitates amoeboid migration along the path of least resistance. Nature 568, 546-550 (2019).

31. Sixt, M. \& Lämmermann, T. In vitro analysis of chemotactic leukocyte migration in 3D environments. Methods Mol. Biol. 769, 149-165 (2011).

32. Kiermaier, E. et al. Polysialylation controls dendritic cell trafficking by regulating chemokine recognition. Science 351, 186-190 (2016).

33. Zhou, Y. et al. Metascape provides a biologist-oriented resource for the analysis of systems-level datasets. Nat. Commun. 10, 1523 (2019).

\section{Acknowledgements}

This study received support from the European Research Council (Starting grant 635872, CIRCODE to C.S.), the Deutsche Forschungsgemeinschaft (DFG; German Research
Foundation) (Emmy-Noether grant SCHE 1645/2-1 and SFB914 projects B09 and Z03 to C.S.), the Swiss National Science Foundation (SNF 310030_182417/1 to C.S.), the Novartis foundation (20A019 to C.S.) and the International Max Planck Research School for Molecular Life Sciences (IMPRS-LS to C.S.). F.S. was supported by an SGED/ SSED Young Investigator grant. Work in the laboratory of C.D. was supported by a Swiss National Science Foundation grant (310030 184708/1), the Vontobel Foundation, the Novartis Consumer Health Foundation, Swiss Life Foundation, the EFSD/Novo Nordisk Program for Diabetes Research in Europe, the Leenaards Foundation, the Olga Mayenfisch Foundation and Fondation pour l'innovation sur le cancer et la biologie. D.V. received support from the DFG (SFB1009, project A1). C.H. gratefully acknowledges financial support from the Swiss National Science Foundation (310030_182528). H.O. received support from the DFG (OS353-7/1 and OS353-10/1). J.R. received support from the Peter Hans Hofschneider Professorship of the Stiftung Experimentelle Biomedizin, the DFG (grant SFB914, project A12) and the LMU Institutional Strategy LMU-Excellent within the framework of the German Excellence Initiative. We thank the Ludwig-Maximilian University flow cytometry core facility, especially L. Richter and the University of Geneva flow cytometry core facility for their services as well as the core facility for animal models (CAM) of the BMC and the animal facility in Geneva. We thank J. Arasa Aparici for helping us set up the GM-CSF hybridoma culture, V. Petrenko for providing BM from Bmall ${ }^{-/-}$animals and J. Ripperger and U. Albrecht for providing BM from Per1 $1^{-/-} / \mathrm{Per}^{-/-}$mice.

\section{Author contributions}

S.J.H. devised, performed, analyzed and interpreted experiments and wrote the paper L.M.I., C.B., M.T.S., F.S., V.P., R.P., S.J., J.W., D.L., J.P.-M. and C.-S.C. performed, analyzed and interpreted experiments. M.M. and W.-H.B. provided human samples. L.H., D.V., M.S., B.U.S., C.H., C.D., H.O. and J.R. provided reagents and provided help in experimental analysis and interpretation. C.S. conceptualized the study, devised, performed, analyzed and interpreted experiments and wrote the paper.

\section{Competing interests}

The authors declare no competing interests.

\section{Additional information}

Extended data is available for this paper at https://doi.org/10.1038/s41590-021-01040-x. Supplementary information The online version contains supplementary material available at https://doi.org/10.1038/s41590-021-01040-x.

Correspondence and requests for materials should be addressed to Christoph Scheiermann.

Peer review information Nature Immunology thanks Beat Imhof, Ana-Maria Lennon-Dumenil and Scott Mueller for their contribution to the peer review of this work. Ioana Visan was the primary editor on this article and managed its editorial process and peer review in collaboration with the rest of the editorial team.

Reprints and permissions information is available at www.nature.com/reprints. 
a

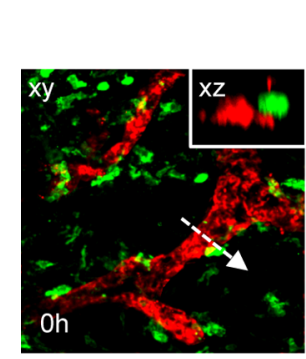

CD11C LYVE-1

C

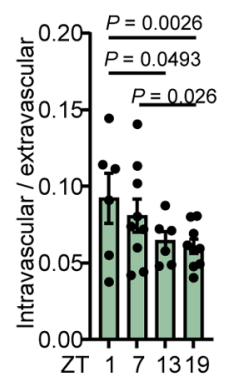

g

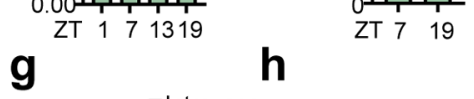

d

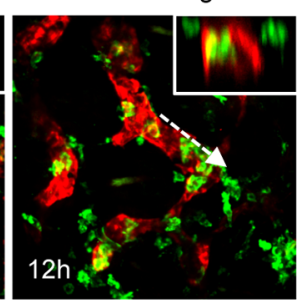

Endogenous $\mathrm{CD} 11 \mathrm{c}^{+}$cells

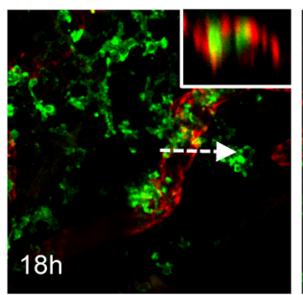

e

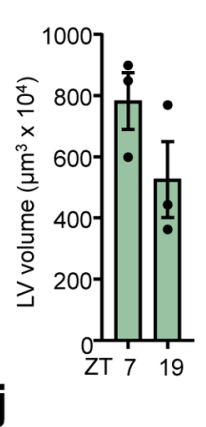

f b
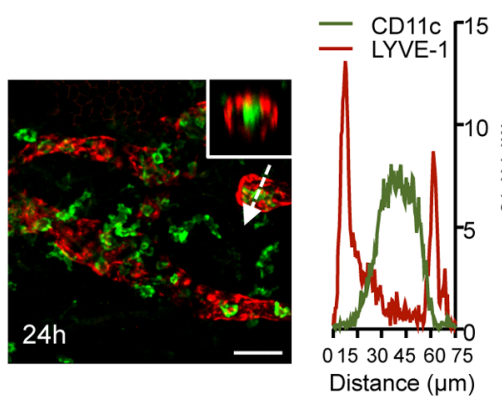

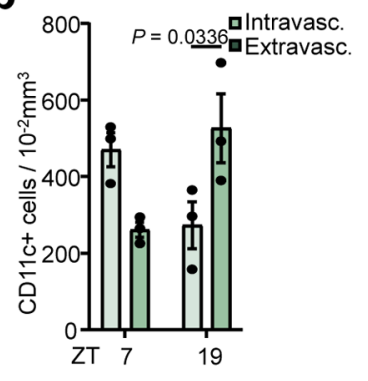

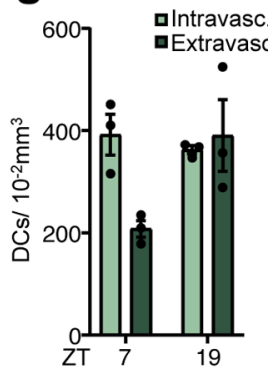
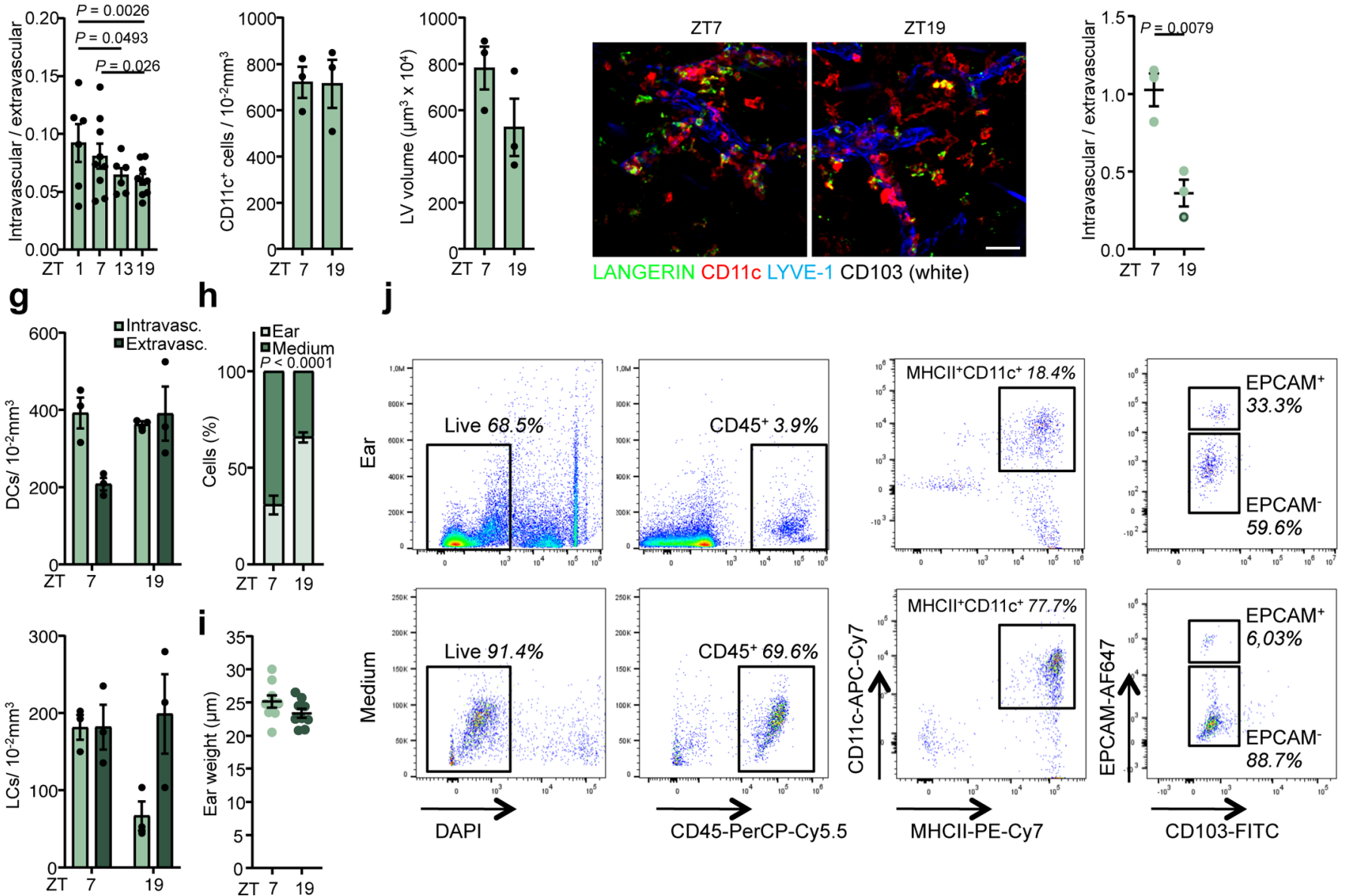

Extended Data Fig. 1 | Endogenous CD11c cell analysis. a-d, Endogenous crawl-in assay with increasing incubation times, intraluminal CD11c ${ }^{+}$cells can be identified in LYVE-1+ lymphatic vessels (LVs) by fluorescence line plot analysis. Scale bar, $50 \mu \mathrm{m}$ and $10 \mu \mathrm{m}$ for orthogonal views, $\mathbf{a}$, representative of 2 independent experiments. $\mathbf{b}$, Representative numbers of CD11c cells within or outside of LVs after a 24 h endogenous crawl-in assay, normalized to LV volume and used to calculate the intravascular versus extravascular ratio, $n=3$ mice, data are representative of 5 independent experiments, two-way ANOVA with Šidák correction. c, Ratio of intravascular versus extravascular CD11c cells, directly after harvest, $n=6,9,6,9$ mice, data are representative of 3 independent experiments; one-way ANOVA with Tukey's post-test. $\mathbf{d}$, Total number of CD11c ${ }^{+}$cells counted per field of view after a $24 \mathrm{~h}$ endogenous crawl-in assay, $\mathrm{n}=3$ mice, data are representative of 5 independent experiments. $\mathbf{e}$, Analysed LV volume per field of view, $\mathrm{n}=3$ mice, data are representative of 5 independent experiments. $\mathbf{f}, 24 \mathrm{~h}$ endogenous assay staining for LANGERIN, CD11c and LYVE-1 to measure the intravascular versus extravascular ratio of Langerhans cells (LCs), unpaired student's $t$-test, scale bar, $50 \mu \mathrm{m}, \mathrm{n}=3$ mice, data and images are representative of 2 independent experiments. $\mathbf{g}$, Representative numbers of DCs and LCs within or outside of LVs after a 24 h endogenous crawl-in assay, $\mathrm{n}=3 \mathrm{mice}$, data are representative of 2 independent experiments. $\mathbf{h}$, Relative numbers of emigrated (medium) or resident (ear) $\mathrm{MHCll}^{+} \mathrm{CD} 11 \mathrm{c}^{+} \mathrm{EPCAM}+\mathrm{LCs}$ after a $24 \mathrm{~h}$ crawl-out assay, measured by flow cytometry, $n=3$ mice, data are representative of 3 independent experiments, two-way ANOVA with Šidák correction. i, Weight of ears used for crawl out assays, $n=9$ mice from 3 independent experiments, two-way ANOVA. $\mathbf{j}$, Gating strategy of EPCAM+/- CD11c ${ }^{+} \mathrm{MHCII}^{+}$ dendritic cells (DCs) resident in ears or emigrated into medium. All data represented as mean \pm s.e.m. 
a

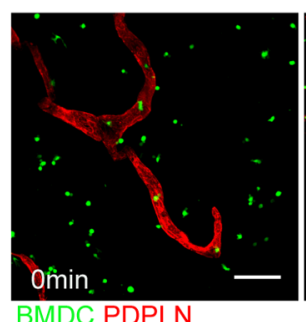

Exogenous BMDCs

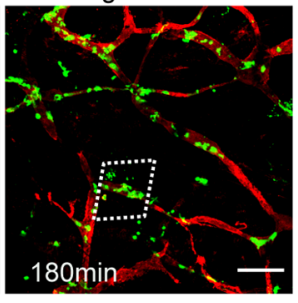

e b

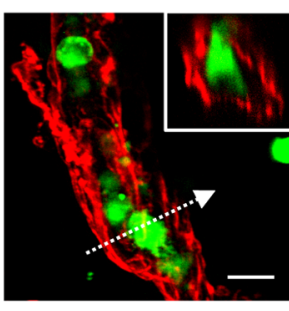

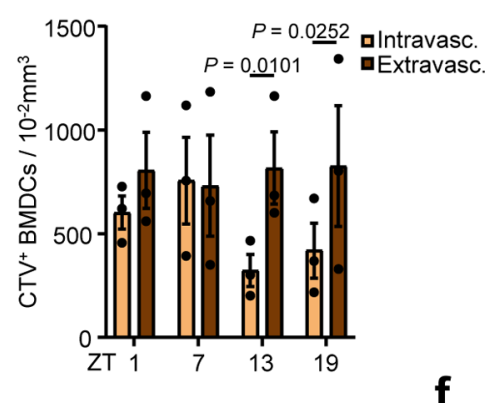

C

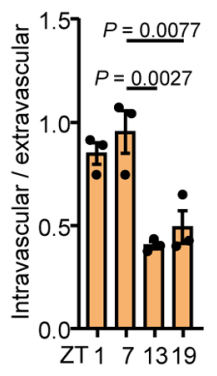

d

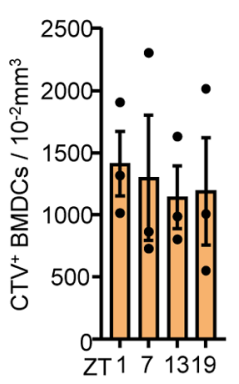

g

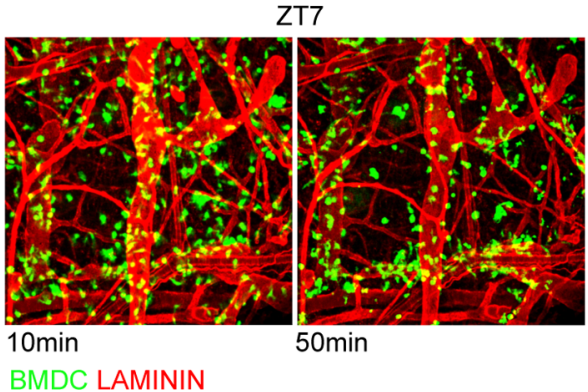

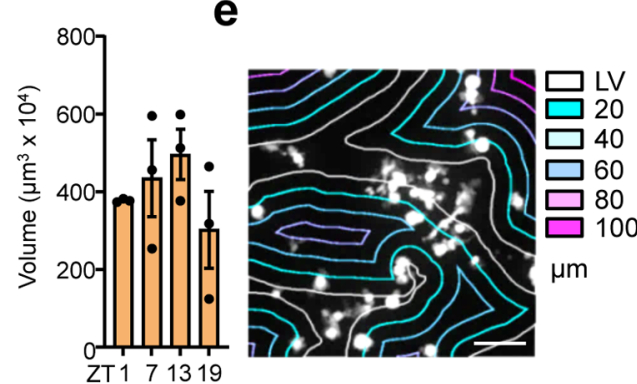

ZT1 71319

T7
ZT19

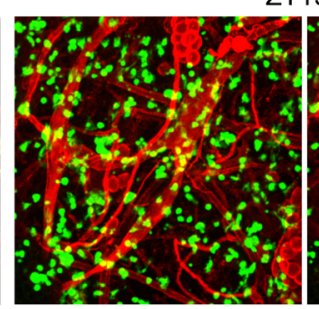

$10 \mathrm{~min}$

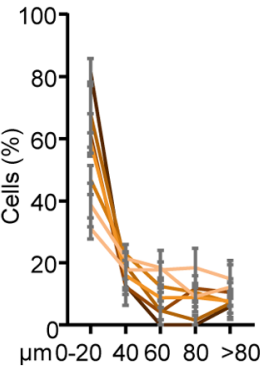

um $0-20406080>80$
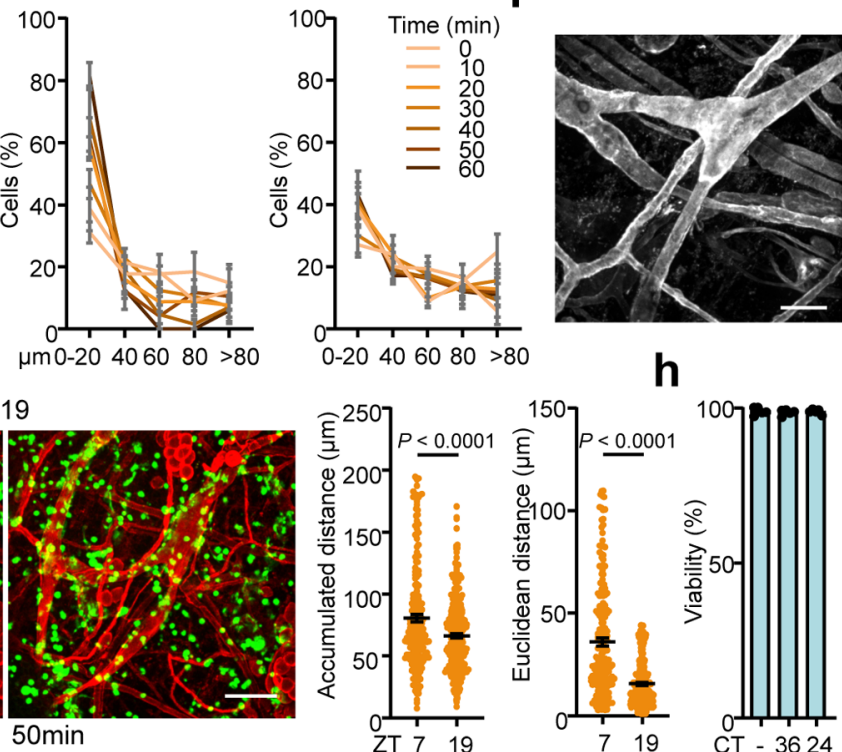

Extended Data Fig. 2 | Exogenous BMDC analysis. a, b, BMDCs of a 3 h exogenous crawl visualized inside the PODOPLANIN+ LV lumen using orthogonal views. Scale bar, $70 \mu \mathrm{m}$ and $10 \mu \mathrm{m}$ for orthogonal views, a. b. Representative numbers of BMDCs within or outside of LYVE-1+ LVs normalized to vessel volume after a $3 \mathrm{~h}$ exogenous crawl in assay, $\mathrm{n}=3$ mice, data are representative of 5 independent experiments. c, Intravascular versus extravascular ratio of individual BMDC batches after a $3 \mathrm{~h}$ incubation time. $\mathbf{d}$, Total count of BMDCs per field of view normalized to LV volume (left) and calculated LV volume (right), one-way ANOVA with Tukey's multiple comparisons test, $n=3$ mice from 4 independent experiments. e, BMDC crawl-in assays with increasing incubation times and analysed by distance dependent zone segmentation of the lymphatic interstitial area, scale bar, $50 \mu \mathrm{m}$. Relative accumulation of BMDCs after 0-60 min incubation shown for Zeitgeber Time (ZT) 7 and 19, $n=4$ mice, data are representative of 2 independent experiments.

f, Representative whole mount staining from 2 independent experiments of the skin for LAMININ used for live cell imaging, scale bar, $50 \mu \mathrm{m}$. $\mathbf{g}$, Live cell imaging of BMDCs administered to split ears harvested at ZT7 or ZT19 for 50 min and quantification of accumulated and euclidean distance, unpaired student's t-test, 220 BMDCs for ZT7, 300 BMDCs for ZT19 from 4 mice from 4 independent experiments, scale bar, $100 \mu \mathrm{m}$. $\mathbf{h}$, Viability of control and circadian time (CT) 36 / CT24 BMDCs after serum shock synchronization measured by flow-cytometry, $\mathrm{n}=3$ donor mice, data are representative from 3 independent experiments. All data are represented as mean \pm s.e.m. 
a
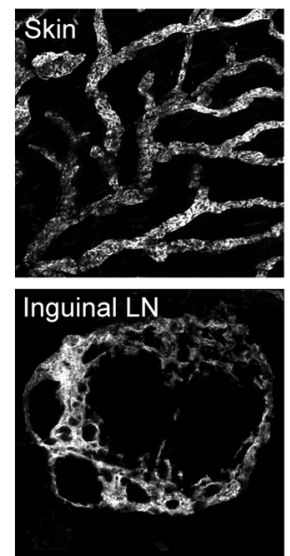

C Lung
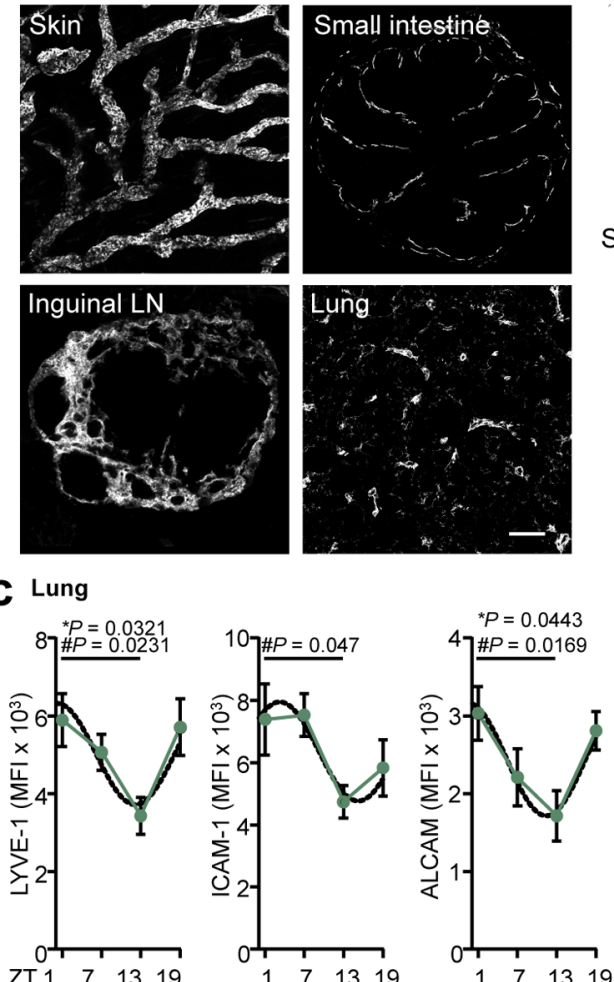

Inguinal lymph node
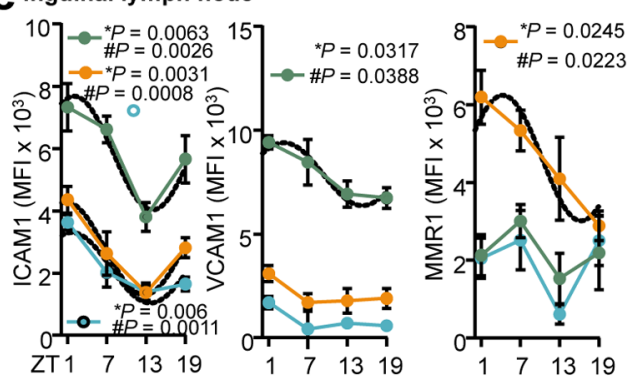

f Small intestine
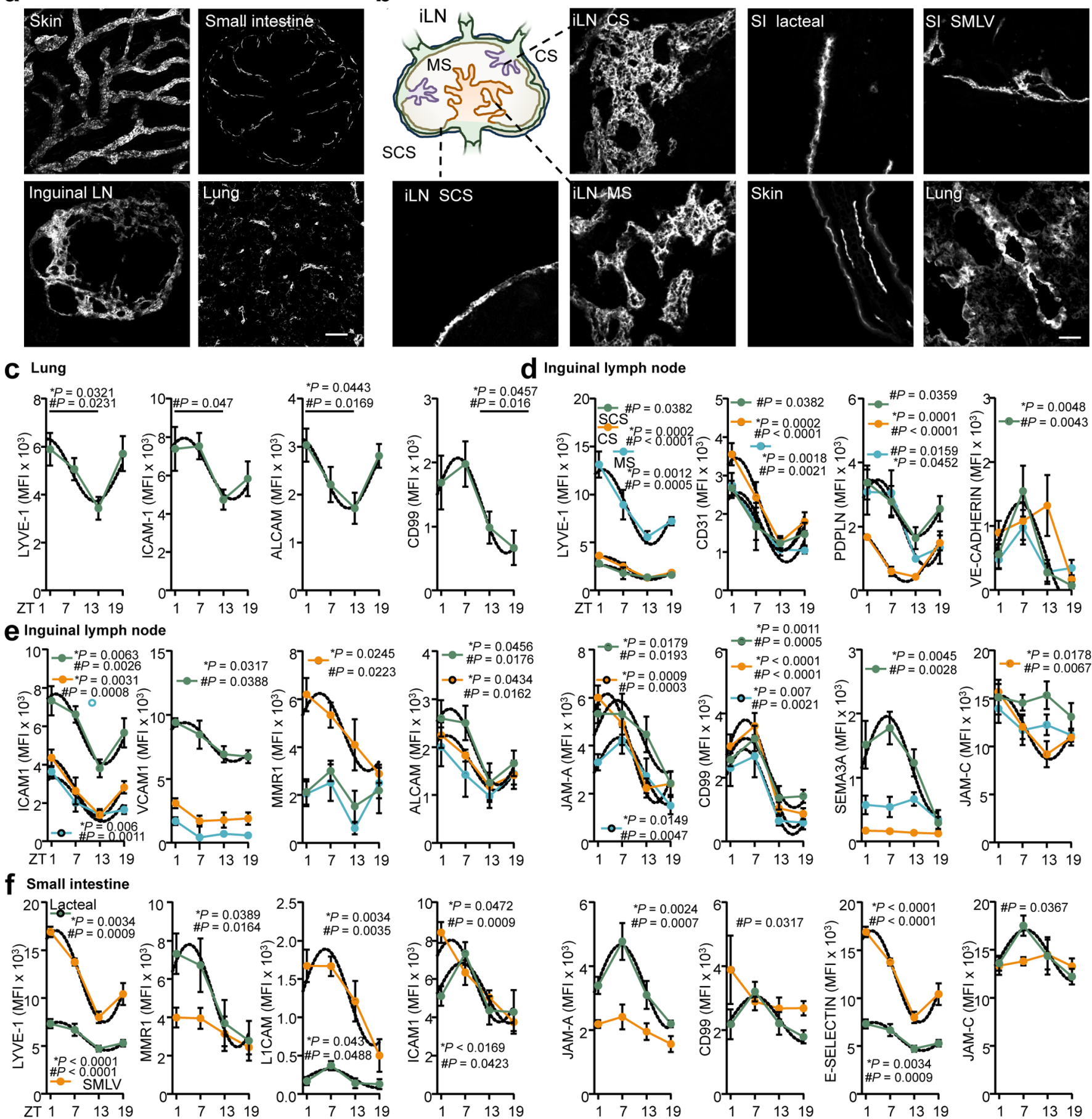

d Inguinal lymph node
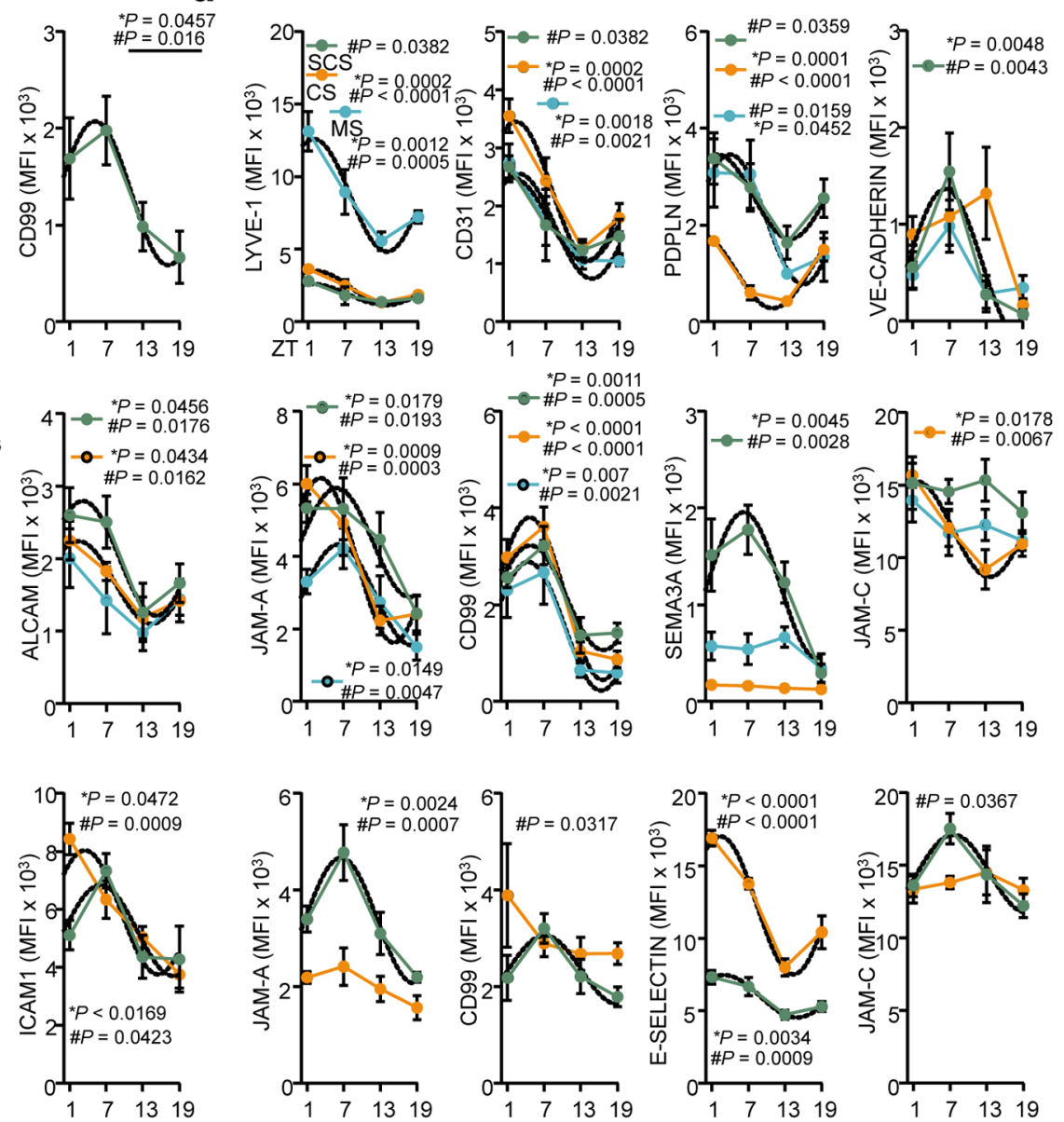

gom
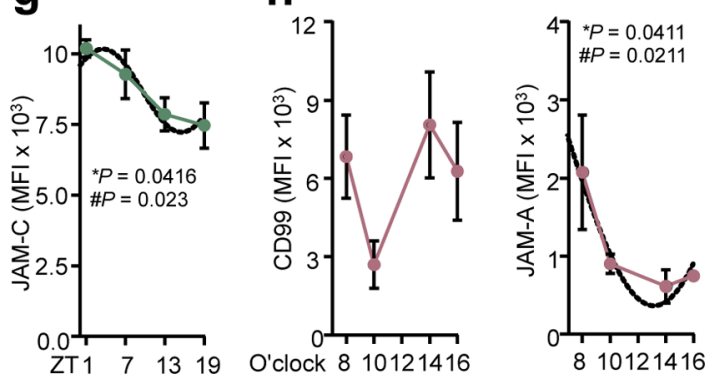

Extended Data Fig. 3 | See next page for caption. 
Extended Data Fig. 3 | LEC protein analysis. a, b, LYVE-1+ staining overviews of skin, small intestine (SI), inguinal lymph node (iLN) and lung tissues used for quantitative analysis. Scale bar, $150 \mu \mathrm{m}, 100 \mu \mathrm{m}$ for skin, representative images of 2 independent experiments, $\mathbf{a}$. b, Respective sub-structures and higher magnifications for analysis. Schematic view of the iLN marks lymphatic sinusoids. CS = cortical sinus, MS $=$ medullary sinus, $S C S=$ subcortical sinus, SMLV = sub-mucosal LV, representative images of 2 independent experiments. Scale bar, $50 \mu \mathrm{m}$. c-g, Quantitative immuno-fluorescence microscopy screen of proteins on LVs in mouse lung, $\mathbf{c}$, iLN, $\mathbf{d}, \mathbf{e}, \mathrm{Sl}, \mathbf{f}$, and skin, $\mathbf{g}$, sections as well as sections from human skin biopsies, $\mathbf{h} .{ }^{*}=0$ one-way ANOVA, \# = cosinor analysis, $n=5$ for murine (data are representative of 2 independent experiments) and 7,12,7,5 (CD99) and 5,8,4,7 (JAM-A) for human samples. Dotted line represents fit curves. All data are represented as mean \pm s.e.m. 
a

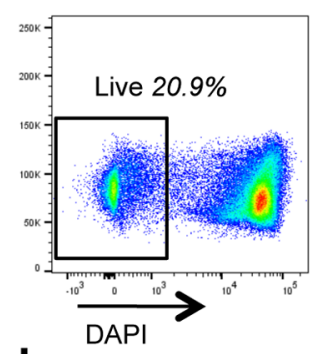

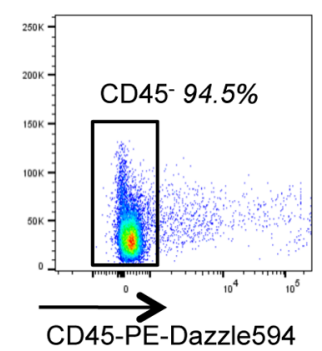

CD45-PE-Dazzle594

d
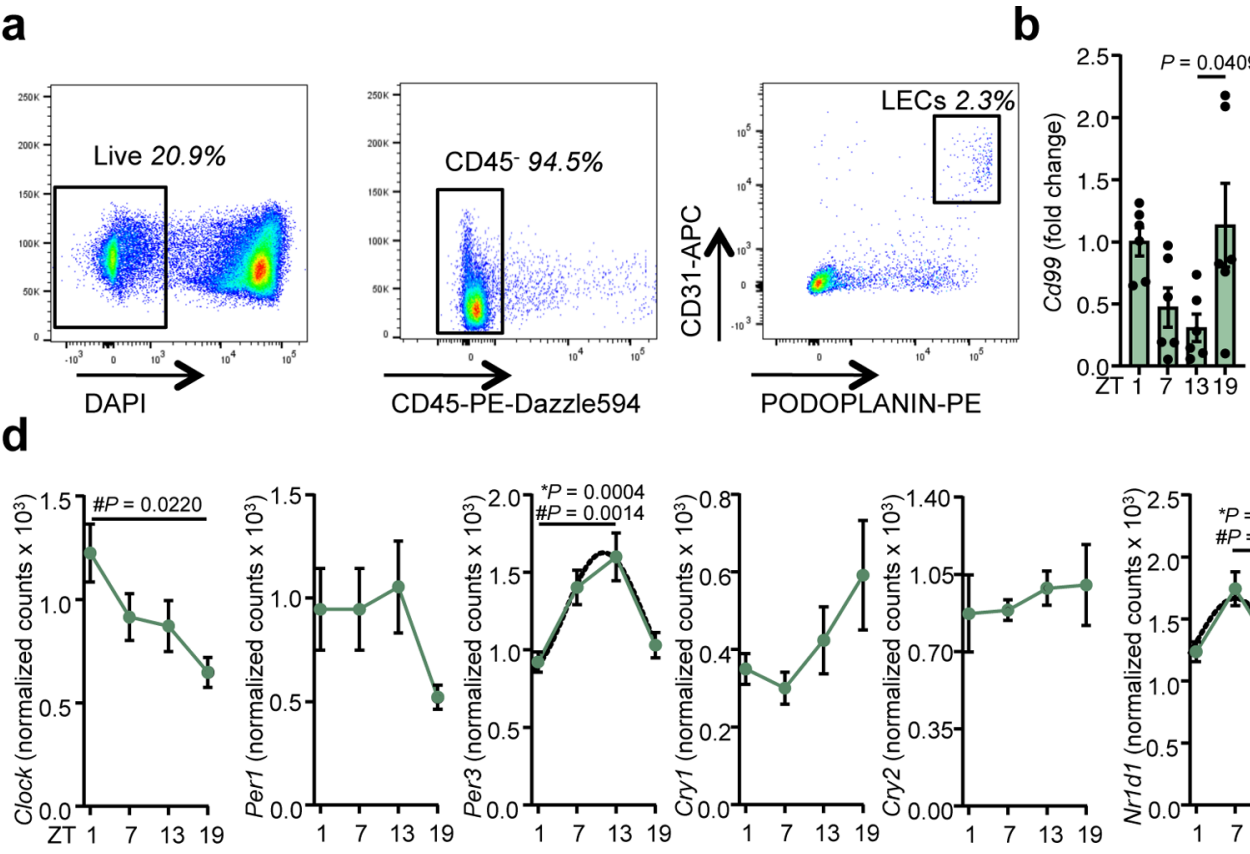

C

C $P<0.0001$

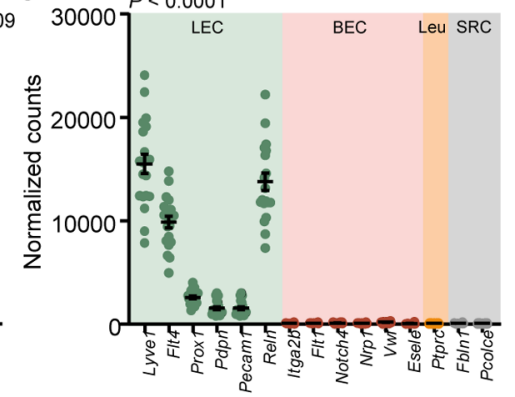

e $\mathrm{f}$
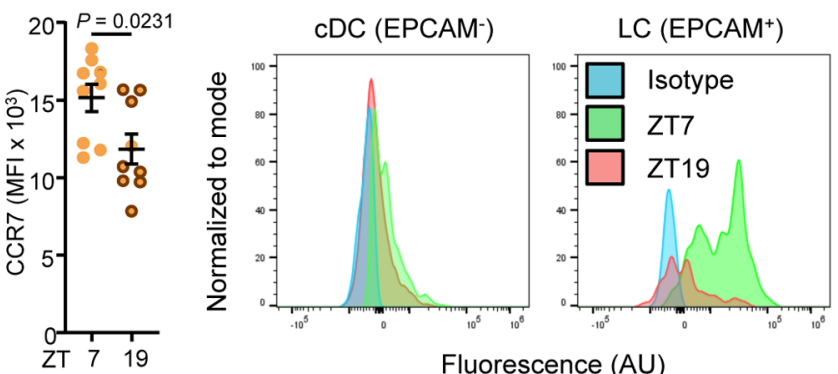

Fluorescence (AU)
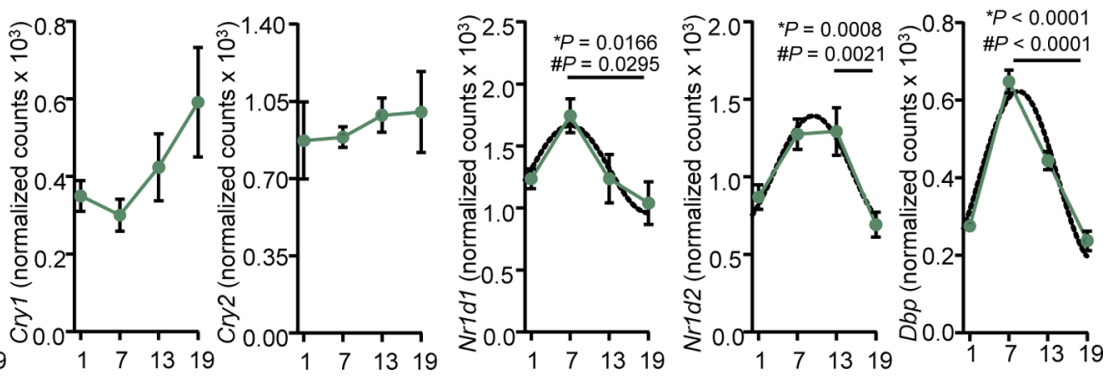

Extended Data Fig. 4 | LEC mRNA and DC protein analysis. a, Gating strategy of sorted dermal CD31+ PODOPLANIN+ lymphatic endothelial cells (LECs) for qPCR and RNA-sequencing analyses. b, Relative mRNA expression of Cd99 in sorted dermal LECs after qPCR analysis, one-way ANOVA with Tukey's post-test, $n=3$ mice, data are representative of 2 independent experiments. $\mathbf{c}$, Expression of $L E C$, blood endothelial cell (BEC), leukocyte (leu) and stromal cell (SRC)-specific genes in sorted dermal LECs presented as normalized counts and analysed by RNA sequencing, one-way ANOVA, $n=20$. d, Clock gene expression of sorted dermal LECs across 4 time points measured and depicted as normalized counts. ${ }^{*}=$ one-way ANOVA, $\#=$ cosinor analysis, $n=$ 5 mice. e, f, Flow-cytometric analysis of CCR7 on emigrated DCs after a $24 \mathrm{~h}$ crawl-out assay. $\mathbf{e}$, MFI of CCR7 on Langerhans cells (LCs), $n=9$ mice from 2 independent experiments. f . Histograms of CCR7 MFI on emigrated CDCs (EPCAM) and LCs (EPCAM+) in comparison to an isotype control, unpaired student's $t$-test. All data are represented as mean \pm s.e.m. 
a

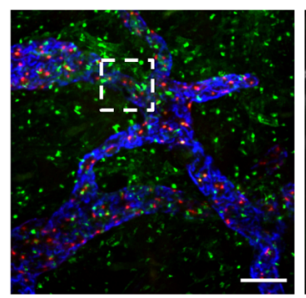

GOLPH4 CCL21

LYVE-1

d

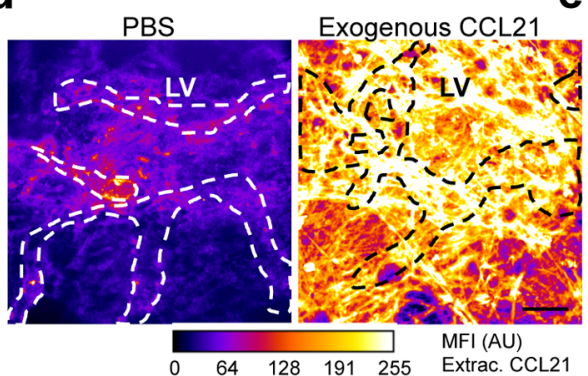

b

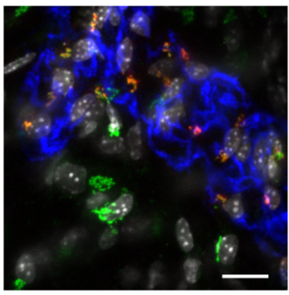

GOLPH4 CCL21 LYVE-1 DAPI (white) b

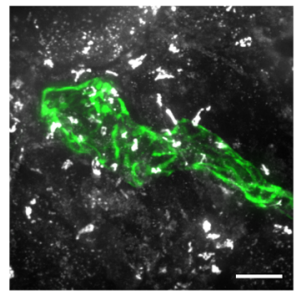

LYVE-1 GOLPH4 (white)

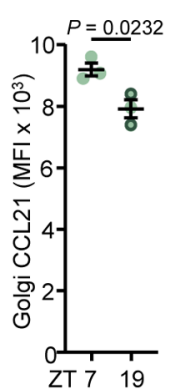

PBS

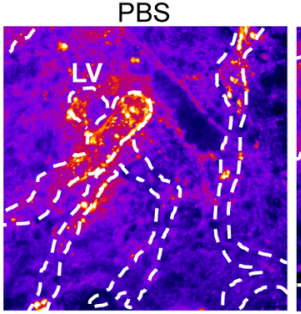

Heparinase

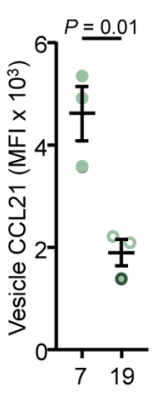

f

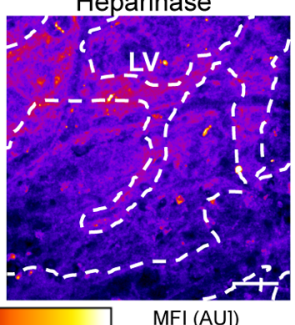

MFI (AUU)

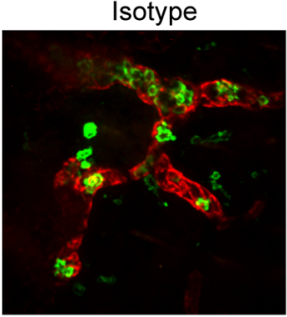

CD11C LYVE-1
C

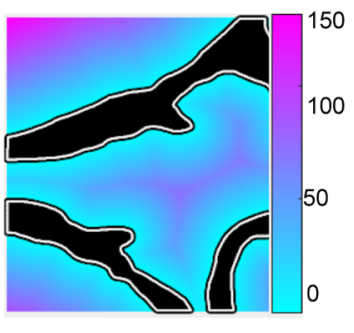

$\mathrm{CCL} 21 \mathrm{Ab}$

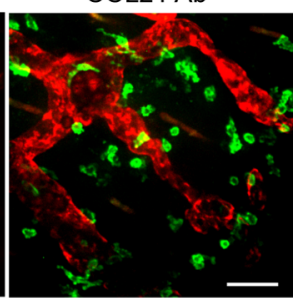

g

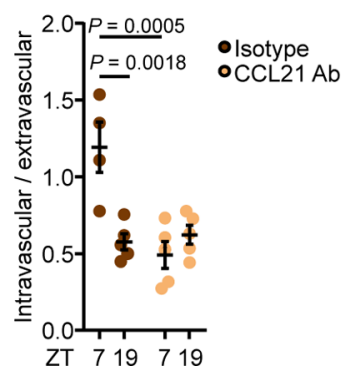

Extended Data Fig. 5 | Chemotaxis analyses. a, Whole-mount staining of CCL21 in GOLPH4+ regions localized in LYVE-1+ capillaries with lower (left) and higher (right) magnification. Scale bar, $50 \mu \mathrm{m}$ and $10 \mu \mathrm{m}$, respectively, representative images of 2 independent experiments. b, MFI of CCL21 in GOLPH4 ${ }^{\text {high }}$ (golgi; left) and GOLPH4 ${ }^{\text {low }}$ (vesicle; right) regions as stained and measured in whole-mounted split ears. Scale bar, $10 \mu \mathrm{m}$, unpaired student's $t$-test, $n=$ 3 mice, data and images are representative of 3 independent experiments. c, Generation of distance maps using LYVE-1+ capillaries for CCL21 gradient analysis. $\mathbf{d}-\mathbf{g}$, Manipulation of CCL21 gradients, images are representative of 2 independent experiments. $\mathbf{d}$, Whole mount staining of non-permeabilized ears for CCL21 after addition of exogenous CCL21. e, Visualization of CCL21 MFI in non-permeabilized ears after heparinase or PBS incubation. Dashed lines represent LYVE-1+ LV outlines. f, $24 \mathrm{~h}$ endogenous crawl-in assay after antibody-mediated blockade of CCL21 in comparison with isotype controls and staining for CD11c and LYVE-1 at ZT7. Scale bars, $50 \mu \mathrm{m} . \mathbf{g}, 3 \mathrm{~h} \mathrm{BMDC}$ crawl-in assay after pharmacological blockade of CCL21, two-way ANOVA with Tukey's post test, $n=4,5,5,5$ mice from 3 independent experiments. All data are represented as mean \pm s.e.m. 
a

b

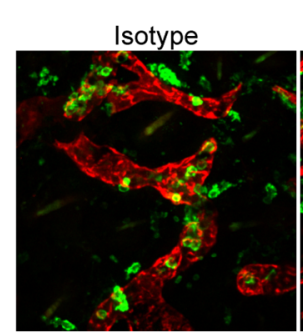

LYVE-1 Ab

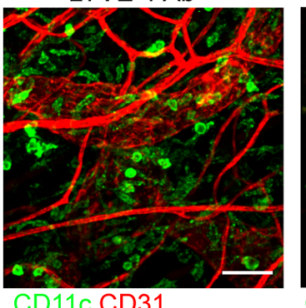

Isotype

C

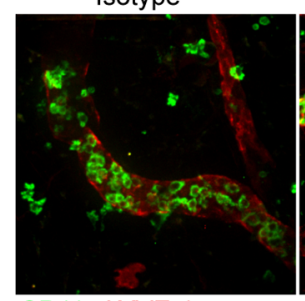

JAM-A Ab

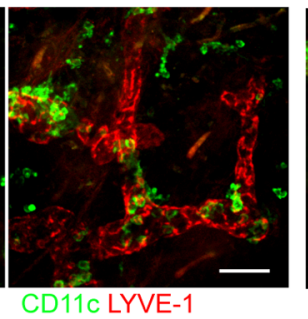

Isotype

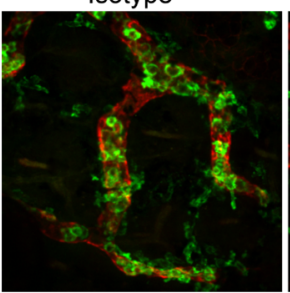

CD99 Ab

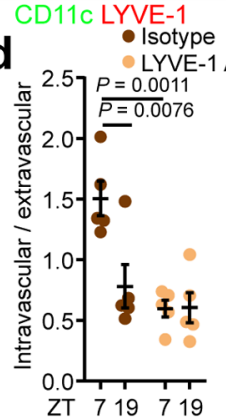

CD11C CD31

CD11C LYVE-1

CD11C LYVE-1

CD11C LYVE-1

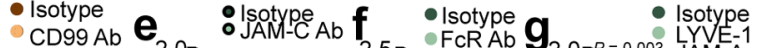
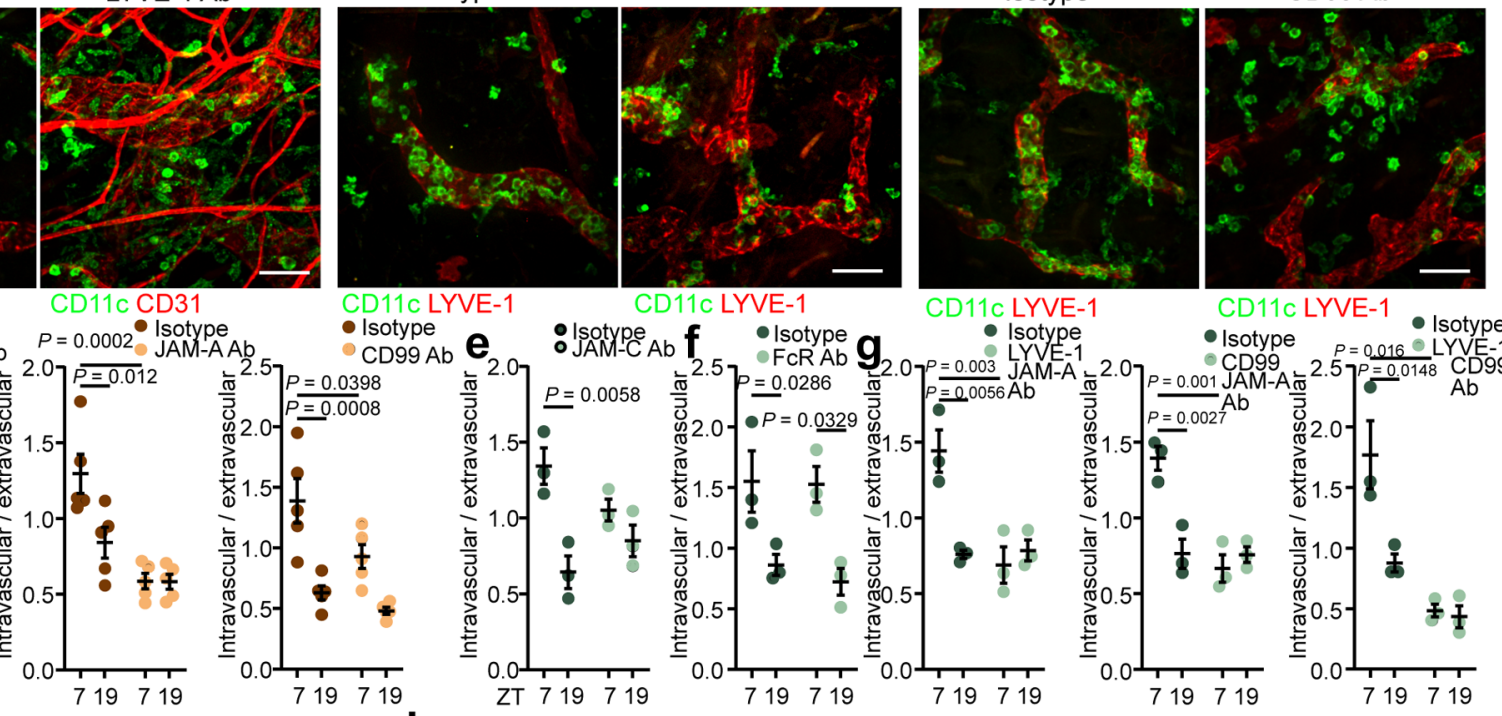

- Isotype

h
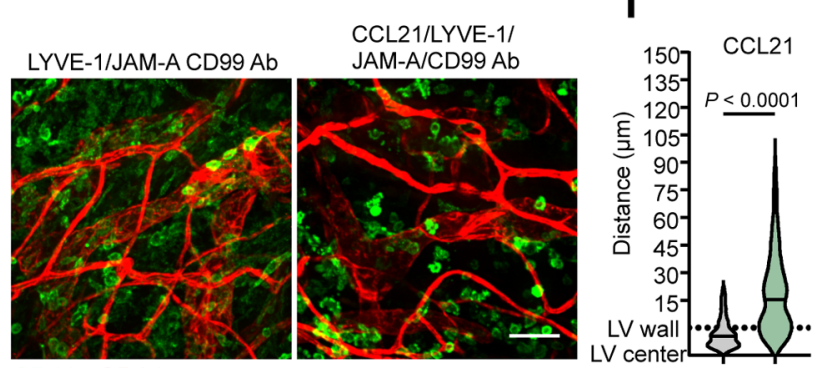

$(-15) \quad$ Ctrl Block
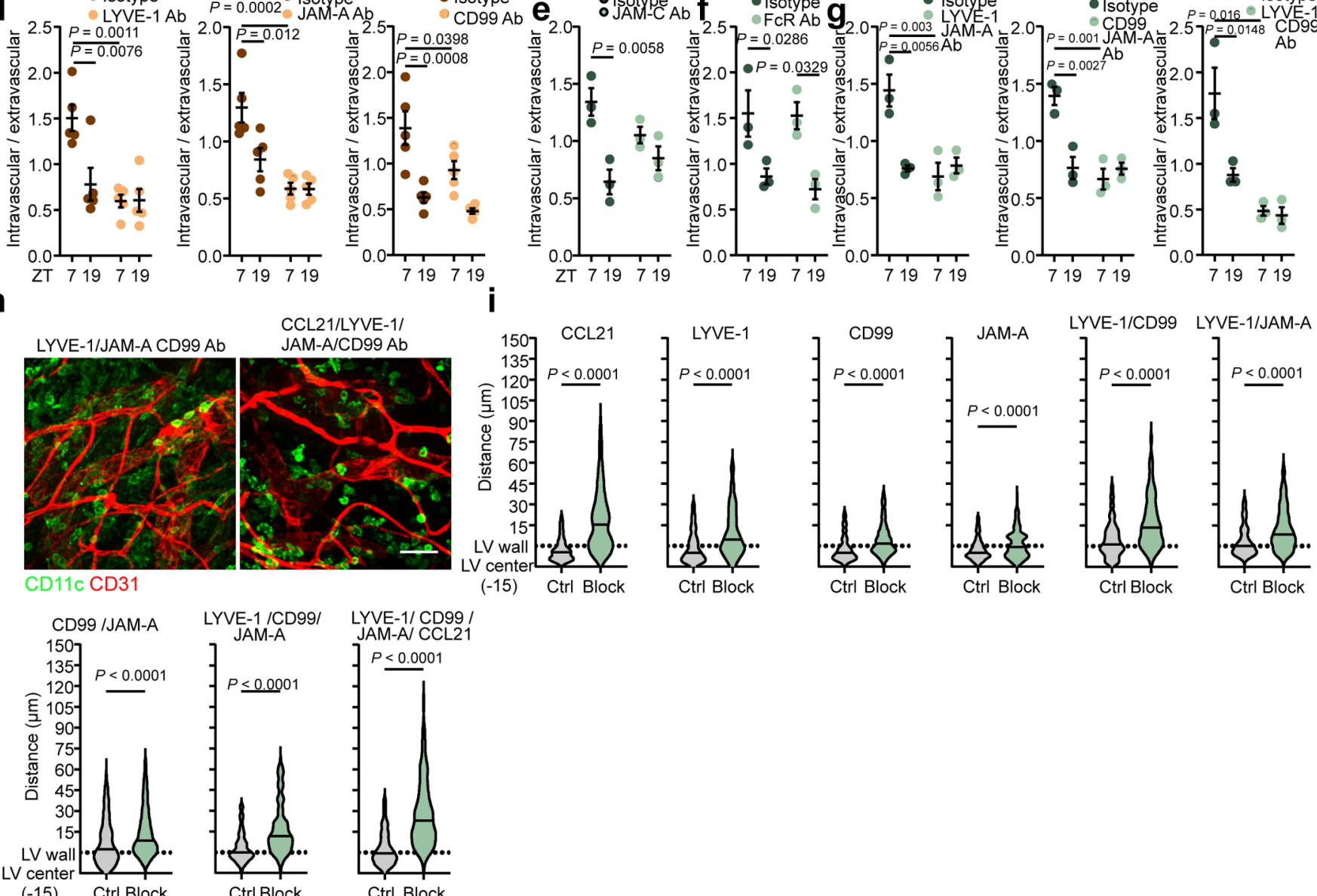

Ctrl Block

Ctrl Block

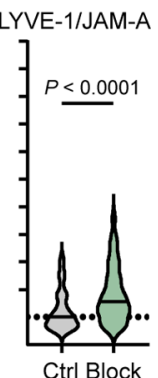

$(-15)$

Ctrl Block

Ctrl Block

Extended Data Fig. 6 | Adhesion and transmigration analysis. a-c, 24 h endogenous crawl-in assays after antibody-mediated blockade of LYVE-1, a, JAM-A, b, or CD99, c, and respective isotype controls, stained for CD11c, CD31 or LYVE-1, representative of 2 independent experiments. Scale bars, 50 $\mu$ m. d, $3 \mathrm{~h} \mathrm{BMDC}$ crawl-in assays after antibody-mediated blockade of LYVE-1 (left), JAM-A (centre) and CD99 (right). $n=5$ mice from 2 independent experiments. e, $\mathbf{f}$, Neutralization of JAM-C (e) and Fc receptor $(F \subset R, \mathbf{f})$ prior to a $24 \mathrm{~h}$ endogenous crawl-in assay with $\mathrm{n}=3$ mice. $\mathbf{g}$, Combinations of anti-LYVE-1, anti-JAM-A and anti-CD99 for double blocks prior to a $24 \mathrm{~h}$ endogenous crawl-in assay, $\mathrm{n}=3$ mice, data are representative of 2 independent experiments, two-way ANOVA with Šidák post test. h, 24 h endogenous crawl-in assay with a combined antibody blockade of LYVE-1, CD99, JAM-A without CCL21 (left) or with CCL21 (right), stained for CD11c and CD31 representative of 2 independent experiments. Scale bar, $50 \mu \mathrm{m}$. i, Distance of $\mathrm{CD}_{11 \mathrm{c}^{+}}$cells to LV centre after $24 \mathrm{~h}$ crawl-in assays and antibody-mediated migration ablation or isotype controls at ZT7. Shown are individual blocks with respective controls, student's $t$-test, $n=364-504$ cells from 3 mice. All data are represented as mean \pm s.e.m. 
a

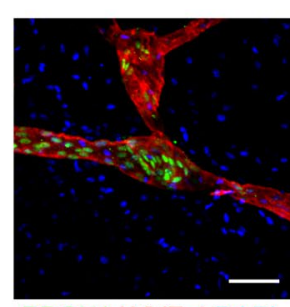

$\mathbf{d}^{\mathrm{PR}}$ b

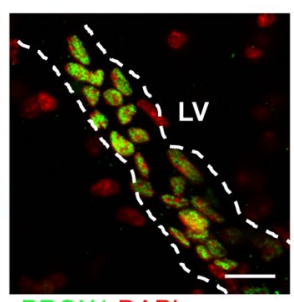

PROX1 DAPI

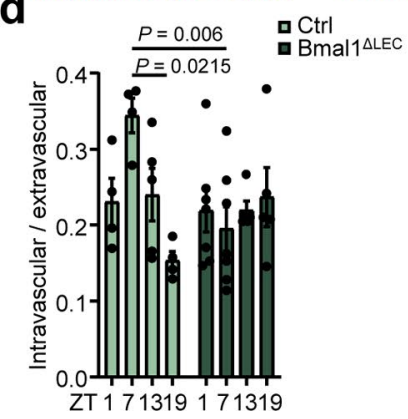

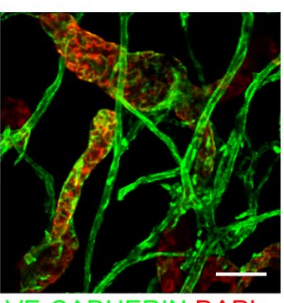

VE-CADHERIN DAPI
C

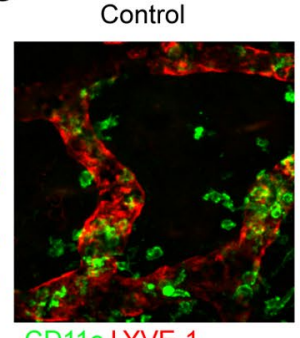

CD11C LYVE-1
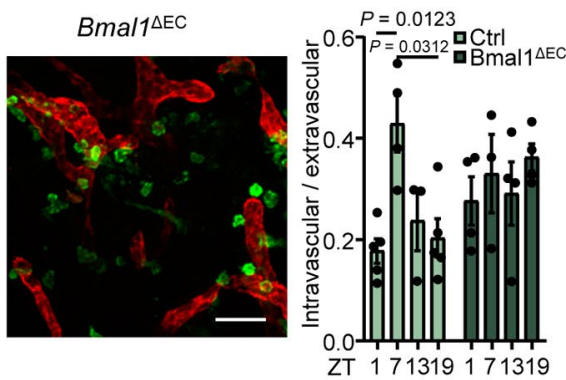

Extended Data Fig. 7 | Lineage-specific BMAL1-deficiency migration analysis. a, b, Representative whole mount staining for PROX-1 and VE-CADHERIN in mouse EAR skin. Dotted lines represent a LYVE- $1^{+}$capillary. Scale bars, $50 \mu \mathrm{m}$ and $10 \mu \mathrm{m}$ for higher magnification. $\mathbf{c}, \mathbf{d}, 6 \mathrm{~h}$ endogenous crawl-in assays

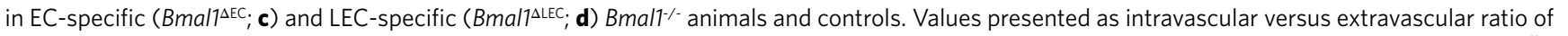
CD11c ${ }^{+}$cells. Representative images show a 24 h endogenous crawl-in assay, stained for CD11c and LYVE-1, scale bar, 50 m, two-way ANOVA with Šidák post test, $\mathrm{n}=(\mathrm{c}, \mathrm{WT})$ 5,4,3,5; (c, KO) 4,3,4,4); (d, WT) 4,4,5,4; (d, KO) 7,7,5,5) mice from 2 independent experiments. All data are represented as mean \pm s.e.m. 
a

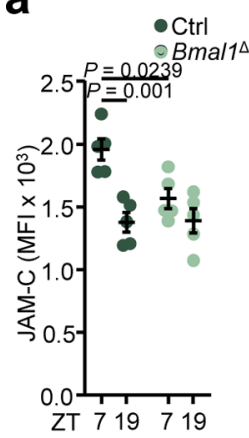

C

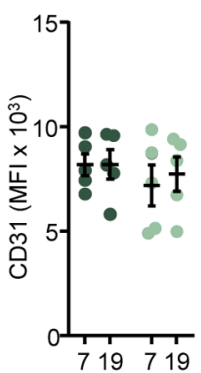

b
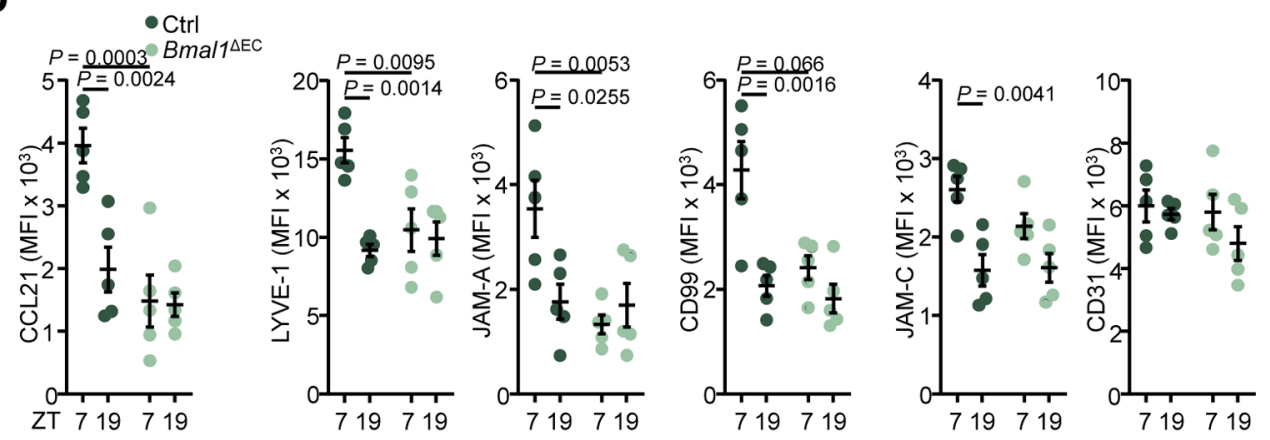

e
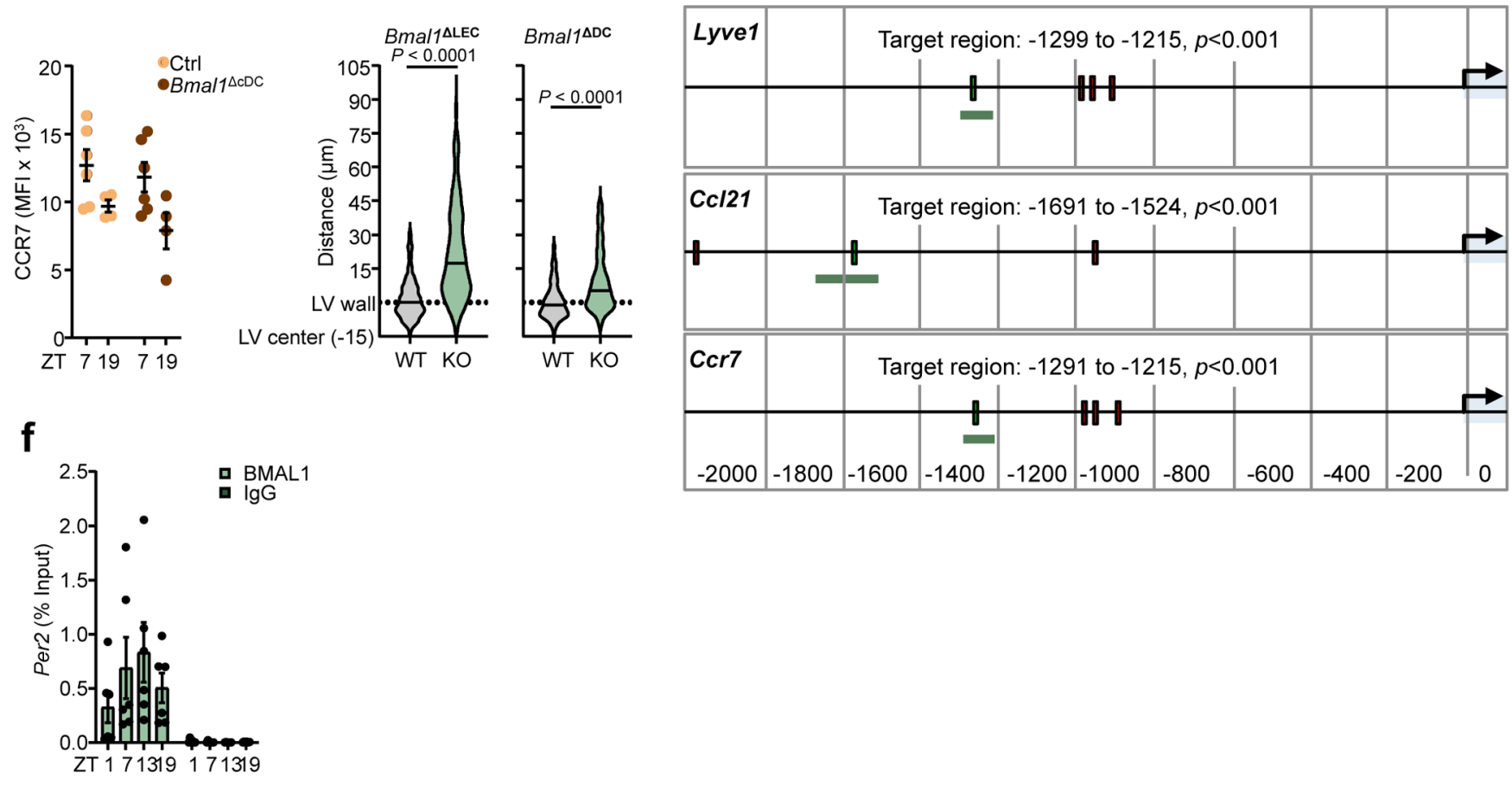

Extended Data Fig. 8 | Lineage-specific BMAL1-deficiency protein analysis and ChIP. a, b, Quantitative immuno-fluorescence microscopy profiling of

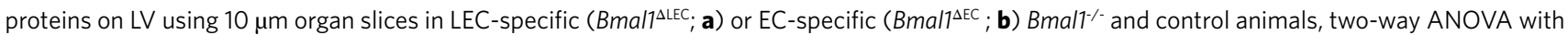
Šidák post test, $n=5$ mice from 2 independent experiments. c, Mean fluorescence intensity (MFI) of CCR7 on the surface of Langerhans cells after a 24

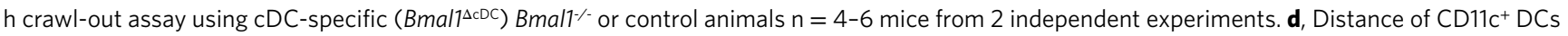
to LV centre after a $24 \mathrm{~h}$ crawl-in assay with tissue-specific Bmal1\%- animals. ${ }^{*}=$ unpaired student's $t$-test, $\mathrm{n}=\left(\right.$ Bmal $\left.{ }^{\mathrm{\Delta LEC}}\right) 371$ and $305 \mathrm{cells}$ from 4 mice; $\left(B m a / 7^{\triangle \mathrm{CDC}}\right) 394$ and 315 cells from 5 mice and 2 independent experiments. e, Schematic overview of promoter regions of target genes Lyve1, Ccl21a and $\mathrm{C} c r 7$ in LN samples for chromatin immunoprecipitation (ChIP) analysis. The $p$-value marks the probability of the green-indicated BMAL1 binding sides, promoter regions are taken from the eukaryotic promoter database, EPFL, Switzerland. f, ChIP qPCR analysis of BMAL1 binding to promoter regions of Per2 as control at different time points throughout the day compared to lgG controls, $n=6$ mice from 2 independent experiments. All data are represented as mean \pm s.e.m. 


\section{Reporting Summary}

Nature Research wishes to improve the reproducibility of the work that we publish. This form provides structure for consistency and transparency in reporting. For further information on Nature Research policies, see our Editorial Policies and the Editorial Policy Checklist.

\section{Statistics}

For all statistical analyses, confirm that the following items are present in the figure legend, table legend, main text, or Methods section.

$\mathrm{n} / \mathrm{a}$ Confirmed

$\bigotimes$ The exact sample size $(n)$ for each experimental group/condition, given as a discrete number and unit of measurement

$\bigotimes$ A statement on whether measurements were taken from distinct samples or whether the same sample was measured repeatedly

$\varnothing$ The statistical test(s) used AND whether they are one- or two-sided

Only common tests should be described solely by name; describe more complex techniques in the Methods section.

Х $\square$ A description of all covariates tested

$\bigotimes$ A description of any assumptions or corrections, such as tests of normality and adjustment for multiple comparisons

$\triangle$ A full description of the statistical parameters including central tendency (e.g. means) or other basic estimates (e.g. regression coefficient)

AND variation (e.g. standard deviation) or associated estimates of uncertainty (e.g. confidence intervals)

For null hypothesis testing, the test statistic (e.g. $F, t, r$ ) with confidence intervals, effect sizes, degrees of freedom and $P$ value noted Give $P$ values as exact values whenever suitable.

Х $\square$ For Bayesian analysis, information on the choice of priors and Markov chain Monte Carlo settings

$\square$ For hierarchical and complex designs, identification of the appropriate level for tests and full reporting of outcomes

$\bigotimes \square$ Estimates of effect sizes (e.g. Cohen's $d$, Pearson's $r$ ), indicating how they were calculated

Our web collection on statistics for biologists contains articles on many of the points above.

\section{Software and code}

Policy information about availability of computer code

Data collection Slidebook 6.0 3i-Intelligent Imaging Innovations; Galaxy, storage platform - LAFUGA Gene Center (LMU); FACSDiva 8.0.1 BD

Data analysis ImageJ / Fiji 1.51n www.imagej.net; Matlab R2018b Mathworks; GraphPad Prism 9.1 GraphPad; Excel 2010/2017 Microsoft; Flowjo 10.4

Flowjo, LLC; Chemotaxis and Migration Tool 2.0 Ibidi

For manuscripts utilizing custom algorithms or software that are central to the research but not yet described in published literature, software must be made available to editors and

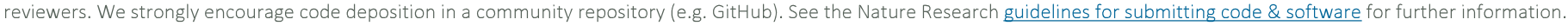

\section{Data}

Policy information about availability of data

All manuscripts must include a data availability statement. This statement should provide the following information, where applicable:

- Accession codes, unique identifiers, or web links for publicly available datasets

- A list of figures that have associated raw data

- A description of any restrictions on data availability

All data that support the conclusions of this paper are available from the authors on request. 
Please select the one below that is the best fit for your research. If you are not sure, read the appropriate sections before making your selection.

\ Life sciences

Behavioural \& social sciences

Ecological, evolutionary \& environmental sciences

For a reference copy of the document with all sections, see nature.com/documents/nr-reporting-summary-flat.pdf

\title{
Life sciences study design
}

All studies must disclose on these points even when the disclosure is negative.

Sample size For initial experimental design, power analyses were performed to determine sample size.

Data exclusions Data was not excluded, unless suggested via statistical testing (GraphPad, Identitication of outliers, ROUT method, Q=1\%) for the real-time imaging analysis only.

Replication All experiments were replicated at least once to prove reproducibility and only included if obtained results were the same.

Randomization Mice were randomly allocated into different experimental groups. Human samples were obtained from patients randomly allocated to different times of the day.

Blinding Blinding was performed for all experiments except the real-time imaging experiments.

\section{Reporting for specific materials, systems and methods}

We require information from authors about some types of materials, experimental systems and methods used in many studies. Here, indicate whether each material, system or method listed is relevant to your study. If you are not sure if a list item applies to your research, read the appropriate section before selecting a response.

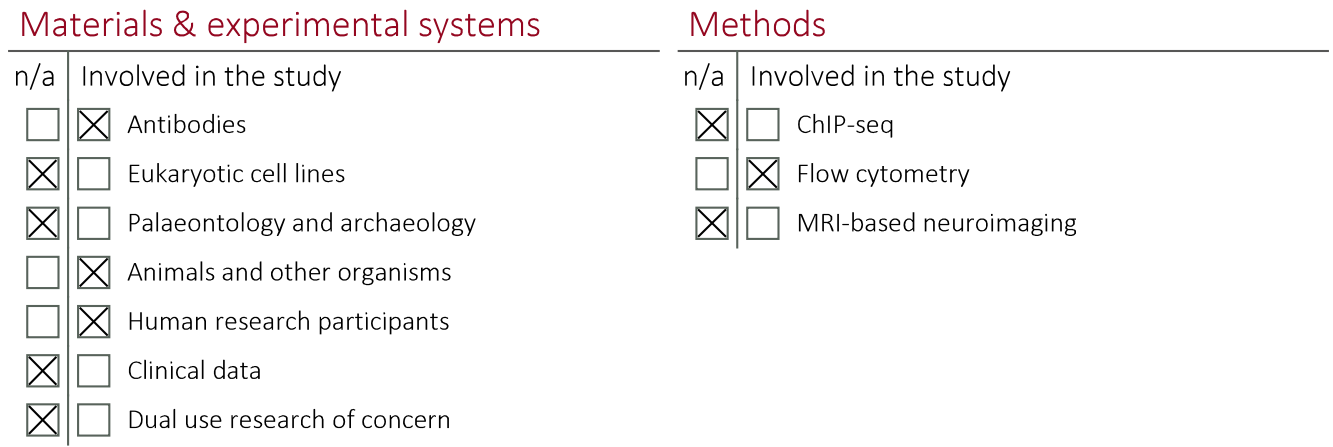

\section{Antibodies}

Antibodies used

A complete list of antibodies including concentrations, fluorophore, clone, reference number, provider and appropriate isotype controls are found within an 'Antibody' table in the supplementary data section.

Validation

\begin{abstract}
All neutralization antibodies used for DC migration were taken from publications that have validated the antibodies prior to this study, including Johnson, L. A. \& Jackson, D. G. Inflammation-induced secretion of CCL21 in lymphatic endothelium is a key regulator of integrin-mediated dendritic cell transmigration. Int Immunol 22, 839-849, doi:10.1093/intimm/dxq435 (2010)); Weber, M. et al. Interstitial dendritic cell guidance by haptotactic chemokine gradients. Science 339, 328-332, doi:10.1126/science.1228456 (2013); Russo, E. et al. Intralymphatic CCL21 Promotes Tissue Egress of Dendritic Cells through Afferent Lymphatic Vessels. Cell Rep 14, 1723-1734, doi:10.1016/j.celrep.2016.01.048 (2016); Johnson, L. A. et al. Dendritic cells enter lymph vessels by hyaluronan-mediated docking to the endothelial receptor LYVE-1. Nat Immunol 18, 762-770, doi:10.1038/ni.3750 (2017); Torzicky, M. et al. Platelet endothelial cell adhesion molecule-1 (PECAM-1/CD31) and CD99 are critical in lymphatic transmigration of human dendritic cells. J Invest Dermatol 132, 1149-1157, doi:10.1038/jid.2011.420 (2012); Cera, M. R. et al. Increased DC trafficking to lymph nodes and contact hypersensitivity in junctional adhesion molecule-A-deficient mice. J Clin Invest 114, 729-738, doi:10.1172/JCI21231 (2004).
\end{abstract}

\section{Animals and other organisms}

Policy information about studies involving animals; ARRIVE guidelines recommended for reporting animal research

Laboratory animals

Male wild-type (WT) C57BL/6N mice aged 6-8 weeks were purchased from either Charles River Laboratories (Sulzfeld, Germany) or Janvier labs (Le Genest-Saint-Isle, France). Cdh5-creERT2 mice (B6) were obtained as a gift from Ralf Adams (Max-Planck-Institute for Molecular Biomedicine Münster, Germany) and crossbred with Bmal1flox/flox (B6) obtained from Jackson Laboratories to be able to target blood (BECS) and lymphatic endothelial cells (LECS). Prox1-creERT2 (B6; purchased from Jackson Laboratories, Bar Harbor, 
Maine, USA) mice were crossbred with Cdh5-creERT2-Bmal1flox/flox to obtain Prox1-creERT2-Bmal1flox/flox mice to specifically target LECs. Cdh5-creERT2 and Prox-1-creERT2 mice were given intraperitoneal tamoxifen injections for five consecutive days at the age of six weeks to induce Cre recombinase expression and excise Bmal1 at the respective flox regions. EYFP; Clec9acre mice were crossbred with Bmal1flox/flox to target conventional dendritic cells (cDCs)28. Cd99-/- mice and bone marrow (BM) cells from Ccr7 /mice Bmal1-/- and Per2::Luc mice were used aged 6-8 weeks. Primary BM cells from Per1/-Per2-/- mice were provided by Jürgen Ripperger and Urs Albrecht (University of Fribourg, Switzerland). All animals were housed under a 12h:12h light:dark (L:D) schedule with ad libitum access to water and food in the Core Facility Animal Models at the Biomedical Centre (LMU, Germany) or the University of Geneva (Switzerland).

Wild animals No wild animals were used in this study.

Field-collected samples No field collected samples were used in this study.

Ethics oversight

All animal procedures and experiments were in accordance with the ministry of animal welfare of the region of Oberbayern and with the German law of animal welfare or were approved and performed in accordance with the guidelines of the animal research committee of Geneva, Switzerland.

Note that full information on the approval of the study protocol must also be provided in the manuscript.

\section{Human research participants}

Policy information about studies involving human research participants

Population characteristics

Skin biopsies were taken from male and female adults (mean age: 74 years).

Recruitment

Skin biopsies were taken from adults presenting at the dermatosurgery unit of the Geneva University Hospitals, Switzerland, for the removal of skin tumours under local anaesthesia. Samples were taken from excessive, tumour-free, surrounding skin known as "Burow's triangles" during the reconstruction of dermatosurgical excisions. No extra incisions were made to obtain the sample and the size of the original tumour excision was not altered by this study. The sample was placed into normal saline solution immediately after it had been excised and the time of day was noted. Samples were then embedded in OCT and shock-frozen until sectioning. No potential self-selection bias or other biases were present.

Ethics oversight

Written informed consent was obtained from each individual. The sampling was conducted according to the Declaration of Helsinki and approved by the Commission Cantonale d'Ethique de la Recherche of the University Hospitals of Geneva.

Note that full information on the approval of the study protocol must also be provided in the manuscript.

\section{Flow Cytometry}

Plots

Confirm that:

\The axis labels state the marker and fluorochrome used (e.g. CD4-FITC).

\The axis scales are clearly visible. Include numbers along axes only for bottom left plot of group (a 'group' is an analysis of identical markers).

\All plots are contour plots with outliers or pseudocolor plots.

\A numerical value for number of cells or percentage (with statistics) is provided.

Methodology

Sample preparation
Crawl-out assays: Harvested and split ears were washed for $2 \mathrm{~h}$ and stored in R10 medium for $24 \mathrm{~h}$ supplemented with $1 \mu \mathrm{g} /$ $\mathrm{ml} \mathrm{CCL21.} \mathrm{Next} \mathrm{day,} \mathrm{ears} \mathrm{were} \mathrm{harvested} \mathrm{and} \mathrm{gently} \mathrm{digested} \mathrm{for} 20 \mathrm{~min}$ at $37^{\circ} \mathrm{C}$ with collagenase IV $(1 \mathrm{mg} / \mathrm{ml}, \mathrm{C} 5138$, Sigma), DNase ( $0.2 \mathrm{mg} / \mathrm{ml}$, Roche) and dispase II $(0.2 \mathrm{mg} / \mathrm{ml}$, Sigma). After digestion, cells were filtered through a $70 \mu \mathrm{m}$ cell strainer, washed and resuspended in PBS supplemented with 2\% FCS and 2 mM EDTA (Sigma). Simultaneously, the medium containing the emigrated DCs was harvested and both the ear DC and medium DC populations were first Fc-receptor blocked with anti-mouse CD16/32 for 5 min at RT and subsequently stained with fluorescence-conjugated antibodies for 30 min at $4^{\circ}$ C. For CCR7 staining, the stain was performed separately at $37^{\circ} \mathrm{C}$ prior to the other staining step. DAPI and full-bright counting beads (Thermo Fisher) were added to cells, which were then analysed by flow-cytometry using a Gallios Flow Cytometer (Beckman Coulter) equipped with a 405, 488 and 633nm laser or BD Fortessa Flow Cytometer (405, 488, 561 and $633 \mathrm{~nm}$ lasers, BD Biosciences).

\section{Sorting of LECS}

To sort dermal LECs for RNA sequencing, 4 ears from two mice were pooled per biological replicate and time point. Dermal cells were isolated as stated above and prepared for sorting on a FACSArialllu (BD) equipped with 4 lasers $(405,488,561,633$ $\mathrm{nm})$ in the Biomedical Centre Munich Core Facility Flow Cytometry. Live PODOPLANIN+ CD31+ LECs were sorted directly into either $350 \mu \mathrm{l}$ TriZol-LS (Thermo Fisher, in case of RNA sequencing) or $350 \mu$ RLT-buffer with $\beta$-ME (1:100) (Qiagen \& Sigma, respectively, in case of qPCR) at $4{ }^{\circ} \mathrm{C}$ using a $100 \mu \mathrm{m}$ nozzle with a purity $>90 \%$ as determined by purity checks after every sort. Cell numbers sorted ranged between 3000 and 5000 cells / two pooled ear pairs. Directly after sorting, samples were shock-frozen on dry-ice and stored for further analysis.

Gallios Flow Cytometer (Beckman Coulter) equipped with a 405, 488 and 633nm laser or BD Fortessa Flow Cytometer (405, 488, 561 and 633nm lasers, BD Biosciences). Sorting: FACSArialllu (BD) 
Software

FACSDiva 8.0.1 BD; Flowjo 10.4 Flowjo, LLC

Cell population abundance

Gating strategy

LEC: Podpoplanin, CD31 (2-3\% final gate)

DC: CD11c, MHCII cells (ear: $15-20 \%$, medium: $70-80 \%$ ) LEC: (2-3\% final gate)

DC: CD11c, MHCII, EPCAM, CD103

W Tick this box to confirm that a figure exemplifying the gating strategy is provided in the Supplementary Information. 